\title{
Basis of the Application of Network Equations to Waveguide Problems
}

\author{
By David M. Kerns
}

\begin{abstract}
A systematic and general formulation of the concepts and the conditions that underlie the technique of the application of network equations to waveguide problems is presented. The discussion is guided by a formulation of what may be called the transducer concept, according to which, essentially, a transducer is a power-transfer device which is to be described only in terms of external characteristics. Waveguide and circuit devices are considered as transducers whose terminal phenomena are electromagnetic fields varying harmonically with time. The basic task is then the definition of suitable terminal variables characterizing the terminal fields. The construction of variables of this kind for waveguide transducers is discussed in considerable detail; for circuits, for which suitable variables are voltage and current, the construction is sketched. Transducer theory is defined, discussed, and illustrated by the development of selected relations of the theory, and is shown to coincide with much of what is generally connoted by "theory of four-terminal networks."

As a matter of interest and importance in applications of the technique, derivations of a rather general reciprocity theorem and of a version of Foster's reactance theorem are included. Both theorems apply to both waveguide and circuit structures.
\end{abstract}

\section{Introduction}

The usefulness of the application of network equations to waveguide problems is rather well known. So-called equivalent circuits have been used since the beginning of intensive work in the microwave field, and they are employed in an increasing number of published papers. This employment of network equations, however, often unnecessarily appears to rest to a considerable degree upon intuition, or upon assumed, rather than proved, analogy with behavior of lowfrequency circuit devices. It is, of course, readily accepted that the procedure can be logically formulated and that analogies can be proved. Valuable discussions have in fact been given by Saxon, ${ }^{1}$ Altar, ${ }^{2}$ and others. Footnote references 1 and 2 both contain, in particular, proofs of reciprocity theorems applying to waveguide structures and thus establish an analogy of the kind mentioned above. But neither of these references is primarily concerned with a general and basic study of the

\footnotetext{
1 Radiation Laboratory, MIT, memorandum, consisting of an introductory section for notes on lectures by Julian Schwinger: Discontinuities in waveguides, prepared by David S. Saxon (Feb. 1945).

guides, prepared by Daviliam Altar, Proc. Inst. Radio Engr., 35, 478 (1947).
}

application of network equations to waveguide problems. Indeed, no general and basic study has appeared, and yet there is an evident need for such a study. The primary purpose of the present paper is to provide a systematic and basic formulation of the technique in question.

The discussion begins with a brief formalization of what may be called the transducer concept, according to which, essentially, a transducer is a power-transfer device which is to be described only in terms of external characteristics. Although the ideas here involved are familiar, it is important that they be specified with reasonable precision and completeness, since the whole aiscussion may be regarded as a development of the application of the transducer concept to a particular class of transducers.

The basic task is thus reduced to the formulation of a method of quantitative description of the external behavior of a class of transducers whose terminal phenomena are electromagnetic fields varying harmonically with time. This class includes both waveguide and conventional circuit devices. In many cases (and only such are con- 
sidered), the terminal electromagnetic fields can be adequately characterized for purposes of transducer theory in terms of a finite number of pairs of variables. The construction of variables of this kind (terminal variables) for waveguide transducers is discussed in considerable detail; for circuits, for which suitable variables are voltage and current, the construction is sketched. Once terminal variables are defined, the characteristics of a given transducer can be quantitatively described in terms of the relationships imposed by the transducer upon its terminal variables.

Transducer theory may be said to be the study of properties of classes of transducers defined by the specification of the mathematical form of the relations connecting the terminal variables. In the present instance, transducer theory so defined coincides with much of what is generally connoted by "theory of four-terminal networks." The method and the meaning of transducer theory from the standpoint of this paper are illustrated by the development of a number of selected relations.

Finally, as a matter of interest and importance in connection with the application of the technique (but not as something fundamental to the technique itself), two theorems are proved: namely, a very general reciprocity theorem similar to one given by Dällenbach, ${ }^{3}$ and a version of Foster's reactance theorem which is somewhat more general than that given by MacLean. ${ }^{4}$ These theorems are given for transducers of either waveguide or circuit type as deductions from Maxwell's equations. In order to prove theorems of this kind it is, of course, necessary to impose suitable hypotheses concerning the interior of the transducers considered. Transducer theory proper, which is exclusively concerned with terminal phenomena, provides an extremely useful framework in which to state results, which may indeed be of either experimental or theoretical origin.

The treatment given here was developed as a part of the material for a course of lectures given at the National Bureau of Standards by the author in the spring of 1947. This paper retains some of the pedagogical aspects of the lecture material. The topic was suggested by Harold Lyons, and the author is grateful to him for suggestions and encouragement in the preparation of the paper.

\footnotetext{
a W. Dallenbach, Der Reziprozitatssat des elektromagnetischen Feldes, Archiv für Elektrotechnik, Bd, 36, Heft 3, 153 (March 1942).

1 W. R. MacLean, Proc. Inst. Radio Engrs., 33, 539 (1945).
}

\section{Transducer Concept}

Since much use will be made of certain general ideas involved in the concept of a transducer, it is desirable to include definitions and a brief discussion of these ideas. A suitable basic definition is that a transducer is "a device actuated by power from one system and supplying power in the same or any other form to a second system." 5 The surfaces (or points) through (or at) which power enters or leaves a transducer will be called terminal surfaces. This term will serve for general purposes, and it anticipates the more specific meaning to be imparted later. The generalization of the basic concept to include transducers with $n$ terminal surfaces is useful and is made here; except perhaps for $n=1$, this generalization is quite acceptable. For $n=1$, the term transducer is not apt, but it is convenient to have this case formally included. The use of the term transducer usually implies a concentration of attention on external characteristics of a device. This aspect of the concept is taken as fundamental and is developed in the following paragraphs.

The description of a transducer, as such, is to be accomplished solely in terms of phenomena occurring at the terminal surfaces; that is, only such quantities come into consideration as are accessible to external measurement. For a given transducer let the variables quantitatively specifying the terminal phenomena, the terminal variables, be denoted by $X_{1}, X_{2}, X_{3}$, ... The characteristics of the transducer are then mathe. matically expressible as the aggregate of the relationships imposed by the transducer upon its terminal variables:

$$
\left.\begin{array}{c}
f_{1}\left(X_{1}, X_{2}, X_{3} \ldots\right)=0, \\
f_{2}\left(X_{1}, X_{2}, X_{3} \ldots .\right)=0 \\
\ldots . . . . . . .
\end{array}\right\}
$$

Sets of equations of this kind, describing the characteristics of a transducer, will be called the equations of the transducer.

One is ordinarily interested not only in the properties of individual transducers, but also in the properties of transducers formed by combination of other transducers. The characteristics of a composite transducer can be calculated from the

\footnotetext{
5 Webster's New International Dictionary, 2d cd., unabridged (G. \& C. Merriam Co., Springfield, Mass., 1934).
} 
characteristics of its individual members if the relations connecting variables of one transducer to variables of another (when the two transducers are joined) are provided. Equations expressing the relations imposed by the joining of two transducers will be called joining equations.

Transducer theory may be defined as the theory of properties of classes of transducers, the classes being defined by the imposition of hypotheses on the mathematical nature of the transducer equations considered, and the joining equations being given. Transducer theory thus yields properties possessed in common by all members of a given class and is distinguished from any theory dealing with particular properties of any individual member of any class, or, more generally, from any theory depending upon hypotheses concerning the inner nature of a transducer.

These ideas have served as a general guide in the organization of the subsequent discussion, and they are more specifically employed (and thus illustrated) in section VI.

\section{Waveguide Transducers}

The term waveguide, as used throughout this paper, denotes those types (and only those types) consisting of either one hollow conductor or two conductors, one of which is hollow and encloses the other. The term therefore includes types of waveguides, such as hollow rectangular pipes, which do not support a principal mode, as well as types, such as coaxial line, which do support a principal mode. A wareguide transducer is formally defined as a transducer that has waveguide leads for its input and output connections. The number of waveguide leads is arbitrary, and the waveguides may individually be of arbitrary cross section. The leads of a waveguide transducer are, by hypothesis, ideal waveguides; that is, waveguides of perfectly cylindrical geometry, made of perfectly conducting, metal, and filled with a medium that is homogeneous, isotropic, nondissipative, and linear. The terminal surfaces of a waveguide transducer are cross-sectional (mathematical) surfaces within the waveguide leads (or within the waveguide leads projected). The interior of a waveguide out to a terminal surface is an integral part of the interior of a waveguide transducer. There is, however, no criterion, axcept that of convenience, for the location of a

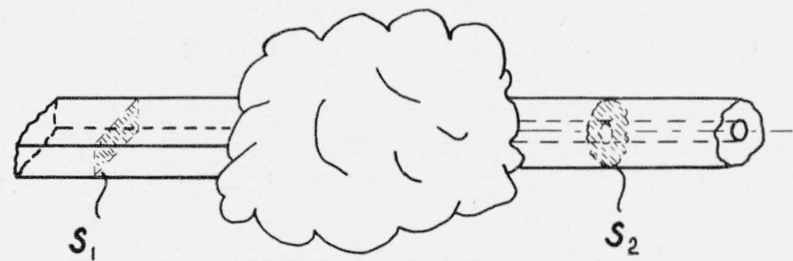

FIgURE 1. Waveguide transducer with two terminal surfaces, $S_{1}, S_{2}$.

terminal surface along a waveguide lead. It will be convenient for purposes of visualization to regard the terminal surfaces as being located within the waveguide leads at some distance from any discontinuity, as indicated in figure 1. A shift in the position of a terminal surface from one given position to another is equivalent to connecting a transducer consisting of the appropriate length of ideal waveguide at the original terminal surface in the wareguide in question; this is a mathematical process, and the details are given at a later point in the discussion (sec. VI).

Examples of waveguide transducers may be found among devices employed in practice in the entire audio-radio spectrum. Such devices may be, for example, attenuators, cavity resonators, transformers, crystal mixers, transmission systems consisting of sending and receiving antennas and intervening space and objects, sections of uniform waveguide, junctions and transitions between two or more waveguides of one or more types, and amplifiers. Provided merely that they possess waveguide leads, such devices are directly admissible to the class of waveguide transducers, regardless of the frequencies for which they may be designed. For practical reasons, the waveguide leads of a low-frequency device must, of course, be of a type that supports a principal mode. The concept of a waveguide transducer is effectively a generalization of the usual concept of a $2 n$-pole (or a $2 n$ terminal network).

The specification of ideal waveguide leads facilitates the mathematical definition of terminal variables which precisely describe the electromagnetic field on a terminal surface; the corresponding physical situation is well-defined, since unshielded leads are excluded. As far as the mathematics is concerned, the subsequent treatment applies unchanged to ideal waveguide consisting of open parallel conductors. But at very high (microwave) frequencies the hypothesis of an 
ideal waveguide of this type is practically untenable. On the other hand, the fact that at sufficiently low frequencies the exact geometry of leads becomes unimportant is familiar, though perhaps not obvious from the standpoint of field theory. An indication of the nature of the approximations involved in the consideration of parallelconductor and ordinary wire leads is given in the latter part of section $\mathrm{V}$.

\section{A Class of Problems of Special Interest}

It is assumed that the field within a waveguide lead varies harmonically with time, with frequency $\omega$. This case is in itself very important in practical problems, but the assumption actually involves no real loss of generality, since an arbitrary time variation may be resolved into sinusoidal components. The time dependence will be represented by the implicit factor exp $(j \omega t)$, and the treatment will thus involve complex amplitudes, rather than instantaneous real quantities. Within a waveguide lead, then, the electric field $\boldsymbol{E}$ and the magnetic field $\boldsymbol{H}$ satisfy Maxwell's equations in the form ${ }^{6}$

$$
\left.\begin{array}{l}
\operatorname{curl} \boldsymbol{E}=-j \omega \mu \boldsymbol{H} \\
\operatorname{curl} \boldsymbol{H}=+j \omega \epsilon \boldsymbol{E}
\end{array}\right\}
$$

where the parameters $\mu, \epsilon$ (representing respectively permeability and dielectric constant) are positive real scalars independent of $\boldsymbol{E}, \boldsymbol{H}$, position, and time $(\mu, \epsilon$ may depend upon $\omega)$. The field is, moreover, subject to the boundary condition that the tangential component of $\boldsymbol{E}$ vanish on the surface of the waveguide.

The most general field satisfying the above differential equations and the boundary condition can be expressed as a sum of an infinite number of elementary particular solutions (modes) characteristic of the cross section of the waveguide. (The fact that the waveguide modes cannot easily be calculated in detail except for a very few simple shapes of cross section is here immaterial. For the purpose of this paper, only general results of the theory of waveguides, holding for waveguides of arbitrary cross section, are needed.) For a given waveguide, and at any given frequency, the number of nonattenuated modes is finite or zero, and the number of attenuated modes is infinite.

\footnotetext{
${ }^{6}$ Rationalized mks, or Giorgi, units are employed throughout.
}

The class of problems primarily to be considered in the text of this paper is now limited as follows: It is assumed that one transducer interacts with another (when they are connected) through the agency of only one waveguide-mode. It is further assumed that this one-mode condition is fulfilled as a consequence of the following more detailed conditions, which correspond to the usual case in practice: (a) the operating frequency is such that one and only one mode can be propagated without attenuation in a waveguide (the propagated mode is then obviously the lowest mode), and (b) the length of waveguide lead is great enough to provide very high attenuation of higher modes. ${ }^{7}$ These conditions insure that the waveguide lead interconnecting two waveguide transducers will act as an effective mode-filter. Regardless of the complexity of the field which may exist at either end of the section of ideal waveguide, the transducers can interact only through the agency of the one nonattenuated mode.

It may happen that the one-mode hypothesis is still applicable, even if a waveguide supports no nonattenuated modes, or more than one nonattenuated mode. But such cases are relatively rare in practice, and will not be considered explicitly. It is, however, of some interest to drop the one-mode hypothesis and to consider any finite number of nonattenuated modes contributing to the interaction " of two waveguide transducers, even though this case is also one seldom encountered. The extension of the subsequent theory to cover this more general case is not difficult; it is given in the appendix.

A given system can be resolved into simpler systems-consisting of waveguide transducers satisfying the one-mode hypothesis in the form adopted-insofar as the given system consists of parts connected (or separated!) by sufficiently long sections of waveguide in which only one mode is propagated without attenuation. The essential point to be observed is that an interconnecting lead by hypothesis is a section of ideal waveguide. This is not to say that an imperfect physicai junction between waveguides cannot be involved; an imperfect junction, like any other discontinuity, belongs in the interior of a waveguide transducer.

${ }^{7}$ It may be said that for any frequency below and not close to a higher-mode cutoff frequency, and for any kind of waveguide, the higher-mode attenuation in a distance of a few times some mean linear measure of the cross section is of the order of $10^{-3}$ or $10^{-4}$ in amplitude. 
It may be noted that the conditions that have been imposed (viz., harmonic time-dependence, and the one-mode hypothesis) are directly concerned only with the situation within a waveguide lead; no condition is directly imposed on the nature of the interior of a waveguide transducer.

\section{Definitions of Terminal Variables}

The task now is, first, to set up suitable definitions of terminal variables, and then to consider the most important properties of these variables as defined. In accordance with the one-mode assumption introduced in section IV, it will be sufficient to consider only one mode-a nonattenuated mode-in specifying the field on a waveguide terminal surface.

It is desirable at this point to set forth briefly results of waveguide theory in sufficient completeness to meet later, as well as immediate, needs. From waveguide theory, it is known that the transverse components $\boldsymbol{E}_{m}, \boldsymbol{H}_{n n}$ of the most general one-mode waveguide field with harmonic timedependence may be written in the following form:

$$
\left.\begin{array}{l}
\boldsymbol{E}_{m}=\left[C_{m} \exp \left(-j \beta_{m} z_{m}\right)+D_{m} \exp \left(j \beta_{m} z_{m}\right)\right] \boldsymbol{E}_{o m} \\
\boldsymbol{H}_{m}=\left[C_{m} \exp \left(-j \beta_{m} z_{m}\right)-D_{m} \exp \left(j \beta_{m} z_{m}\right)\right] \boldsymbol{H}_{o m}
\end{array}\right\}
$$

where

$$
\boldsymbol{E}_{o m}=Y_{m}^{-1 / 2} \boldsymbol{F}_{o m}, \boldsymbol{H}_{o m}=Y_{m}^{+1 / 2} \boldsymbol{k}_{m} \times \boldsymbol{F}_{o m} .
$$

In these expressions, the subscript $m$ denotes the waveguide considered; the coordinate $z_{m}$ and the unit vector $\boldsymbol{k}_{m}$ are parallel to the cylindrical surface of the waveguide, with positive sense directed into the transducer. $\beta_{m}, Y_{m}, \boldsymbol{F}_{\text {om }}$ are, respectively, phase constant, wave-admittance, and electric-field function characteristic of the mode involved. $C_{m}, D_{m}$ are arbitrary complex amplitudes (at $z_{m}=0$ ) of the traveling-wave components of the field traveling in the positive and negative $z_{m}$-directions, respectively. The phase constant $\beta_{m}$ and the wave-admittance $Y_{m}$ in general depend upon the frequency $\left(\omega_{m}\right)$, the constants of the medium $\left(\mu_{m}, \epsilon_{m}\right)$, the geometry of the cross section, and the mode involved; for principal modes, however, $\beta_{m}=\omega_{m} \sqrt{\mu_{m} \epsilon_{m}}$, $Y_{m}=\sqrt{\epsilon_{m} / \mu_{m}}$. For all nonattenuated modes, $\beta_{m}$ and $Y_{m}$ are real and positive. The vector $\boldsymbol{F}_{o m}$ lies in a transverse plane and is a function of position in the transverse plane, but not of $z_{m}$; the form of $\boldsymbol{F}_{o m}$ depends upon the geometry and upon the mode involved. The field equations leave $\boldsymbol{F}_{\text {om }}$ undetermined to the extent of a constant multiplier. $\boldsymbol{F}_{\text {om }}$ is then uniquely determined (apart from sign) by the convenient normalization

$$
\int_{S_{m}} \boldsymbol{F}_{o m}^{2} d S=1
$$

the integral being taken over the cross section $S_{m}$ of the waveguide; eq 5.3 requires in particular that $\boldsymbol{F}_{o m}$ be real. $\quad \boldsymbol{F}_{o m}$ is one member of the set of mutually orthogonal functions for the different modes in the $m^{\text {th }}$ waveguide.

It is now a simple matter to define the terminal variables. It will be worth while to consider definitions of two alternative pairs of variables. The terminal surface is for convenience specifically taken as a plane cross sectional surface; the tangential components of the field on this surface are then $\boldsymbol{E}_{m}, \boldsymbol{H}_{m}$, as given by eq 5.1 for the particular $z_{m}$-plane in which the terminal surface lies. The possible values of $\boldsymbol{E}_{m}, \boldsymbol{H}_{m}$ on the $m^{\text {th }}$ terminal surface may be expressed in terms of quantities $V_{m}, I_{m}$ by means of the equations

$$
\left.\begin{array}{l}
\boldsymbol{E}_{m}=V_{m} \gamma_{o m}^{-1 / 2} \boldsymbol{E}_{o m}, \\
\boldsymbol{H}_{m}=I_{m} \gamma_{o m}^{1 / 2} \boldsymbol{H}_{o m} ;
\end{array}\right\}
$$

or, alternatively, in terms of quantities $A_{m}, B_{m}$ by means of the equations

$$
\left.\begin{array}{l}
\boldsymbol{E}_{m}=\gamma_{o m}^{-1 / 2}\left(A_{m}+B_{m}\right) \boldsymbol{E}_{o m}, \\
\boldsymbol{H}_{m}=\gamma_{o m}^{-1 / 2}\left(A_{m}-B_{m}\right) \boldsymbol{H}_{o m},
\end{array}\right\}
$$

where $\gamma_{o m}^{1 / 2}$ denotes the positive root of a positive real number to be chosen at convenience. ${ }^{8}$ These equations formally define the terminal variables $V_{m}, I_{m}$ and $A_{m}, B_{m}$. $V_{m}$ and $I_{m}$ are complex amplitudes respectively measuring the total tangential electric and magnetic components of the physically determined field on the terminal surface. $A_{m}$ and $B_{m}$ are complex amplitudes respectively measuring the incident and emergent travelling-wave components of the physically determined field on the terminal surface. The

8 The factor $\gamma_{o m}$ inserted in eq 5.4 will appear as a characteristic impedance (see p. 521). Greater generality with respect to the choice of multiplicative factors is permissible (but not useful); for example, arbitrary and independent complex constants could be inserted in the definitions of $V_{m}$ and $I_{m}$. The form adopted for the definition of $A_{m}, B_{m}$ is merely one of several equally convenient possibilities. For example, a factor $\gamma_{\text {oms }}^{1 / 2}$ instead of $\gamma_{o m}^{-1 / 2}$ could be inserted. 
dependence of $V_{m}, I_{m}$ and $A_{m}, B_{m}$ on $z_{m}$, which is apparent upon comparison of the defining eq 5.4, 5.5 with eq 5.1, is not indicated explicitly, since the terminal surface is ordinarily considered to be in a fixed position. The pair $V_{m}, I_{m}$ and the pair $A_{m}, B_{m}$ are obviously related to each other by the equations

$$
\left.\begin{array}{cc}
V_{m}=A_{m}+B_{m}, & 2 A_{m}=V_{m}+\gamma_{o m} I_{m}, \\
\gamma_{o m} I_{m}=A_{m}-B_{m}, & 2 B_{m}=V_{m}-\gamma_{o m} I_{m} .
\end{array}\right\}
$$

Either one of the pairs could be defined in terms of the other by means of these equations. The expressions for power and the equations for the interconnection of two waveguide transducers in terms of these variables are of essential interest and are easily obtained.

Consider the complex power $W_{m}$ supplied to the transducer at the $m^{\text {th }}$ terminal surface. This power is given by the integral of the inward normal component of the complex Poynting's vector over the terminal surface

$$
W_{m}=\frac{1}{2} \int\left(\boldsymbol{E}_{m} \times \boldsymbol{H}_{m}^{*}\right) \cdot \boldsymbol{k}_{m} d S,
$$

where $\boldsymbol{H}_{m}^{*}$ is the complex conjugate of $\boldsymbol{H}_{m}$. The real part of $W_{m}$ is the time average of the instantaneous input power, and the imaginary part of $W_{m}$ is the amplitude of the reactive power exchange across the terminal surface. Employing the defining eq 5.4 for $V_{m}, I_{m}$, the definitions 5.2 of $\boldsymbol{E}_{o m}, \boldsymbol{H}_{o m}$, and the normalizing condition 5.3, one finds

$$
W_{m}=\frac{1}{2} V_{m} I_{m}^{*}
$$

For $A_{m}, B_{m}$, one finds via either eq 5.5 or 5.6 ,

$$
W_{m}=\frac{1}{2 \gamma_{o m}}\left[\left(\left|A_{m}\right|^{2}-\left|B_{m}\right|^{2}\right)-\left(A_{m} B_{m}^{*}-A_{m}^{*} B_{m}\right)\right] .
$$

In this form the real and the imaginary parts are exhibited separately. The presence of the factor $1 / 2$ on the right-hand sides of eq 5.8, 5.9 means that $V_{m}, I_{m}$ and $A_{m}, B_{m}$ as defined are to be interpreted as peak (rather than as root-mean-square) amplitudes.

The mathematics of the interconnection of waveguide transducers is handled by considering that a terminal surface (No. 1, say) of one trans- ducer coincides with a terminal surface (No. 2, say) of another. For the time being, it is assumed that the terminal surfaces coincide at a point within the interconnecting lead at some distance from any discontinuity, as indicated in figure 2 .

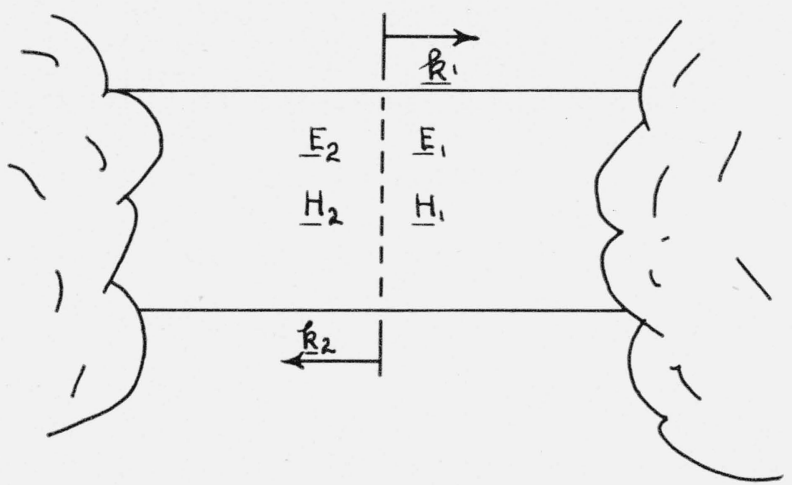

Figure 2. Coincident terminal surfaces.

With the common terminal surface so located, the actual field on the surface may be assumed to be very nearly the field of the one nonattenuated mode. The transverse components of the field on the surface may then be described by the alternative pairs of equations

$$
\begin{aligned}
& \boldsymbol{E}_{1}=V_{1} \gamma_{o 1}^{-1 / 2} \boldsymbol{E}_{o 1}, \text {, } \\
& \left.\boldsymbol{H}_{1}=I_{1} \gamma_{o 1}^{1 / 2} \boldsymbol{H}_{o 1}, \quad\right\} \\
& \boldsymbol{E}_{2}=V_{2} \gamma_{o 2}^{-1 / 2} \boldsymbol{E}_{02}, \text {, } \\
& \left.\boldsymbol{H}_{2}=I_{2} \gamma_{o 2}^{1 / 2} \boldsymbol{H}_{o 2}, \quad\right\}
\end{aligned}
$$

which apply respectively to the two sides of the surface. In order that the whole field (normal and tangential components) be continuous across the mathematical surface, it is sufficient (as well as necessary) that the transition of the tangential components of $\boldsymbol{E}, \boldsymbol{H}$ be continuous at all points of the surface. Thus eq 5.10 and 5.11 must represent identically the same field:

$$
\begin{aligned}
& \boldsymbol{E}_{1} \equiv \boldsymbol{E}_{2}, \\
& \boldsymbol{H}_{1} \equiv \boldsymbol{H}_{2} .
\end{aligned}
$$

Using eq 5.2, these equations become

$$
\begin{gathered}
V_{1} \boldsymbol{F}_{o 1}\left(\gamma_{o 1} Y\right)^{-1 / 2} \equiv V_{2} \boldsymbol{F}_{o 2}\left(\gamma_{o 2} Y_{2}\right)^{-1 / 2}, \\
I_{1} \boldsymbol{k}_{1} \times \boldsymbol{F}_{o 1}\left(\gamma_{o 1} Y_{1}\right)^{1 / 2} \equiv I_{2} \boldsymbol{k}_{2} \times \boldsymbol{F}_{o 2}\left(\gamma_{o 2} Y_{2}\right)^{1 / 2} .
\end{gathered}
$$

Waveguide 1 and waveguide 2 are electrically identical; hence $Y_{1}=Y_{2}$ and $\boldsymbol{F}_{o 1}= \pm \boldsymbol{F}_{02}$. Assum- 
ing that $\boldsymbol{F}_{01}$ and $\boldsymbol{F}_{02}$ are chosen to have the same sign, the equations expressing the fact that the two transducers are joined become

$$
\begin{aligned}
V_{1} & =N_{12} V_{2}, \\
-I_{1} & =I_{2} / N_{12},
\end{aligned}
$$

where $N_{12}=\left(\gamma_{o 1} / \gamma_{o 2}\right)^{1 / 2}$.

The expression of the same fact in terms of $A_{1}$, $B_{1}, A_{2}, B_{2}$ is

$$
\begin{aligned}
& N_{12} A_{2}=B_{1}, \\
& N_{12} B_{2}=A_{1} .
\end{aligned}
$$

Since the value of $\gamma_{o m}$ is arbitrary, and since waveguide 1 and 2 are electrically identical, it is clearly convenient and sensible to have $\gamma_{o 1}=\gamma_{o 2}$; otherwise an apparent discontinuity appears at a place where there is no physical discontinuity at all. If $\gamma_{o 1}=\gamma_{o 2}$, then $N_{12}=1$, and the above equations reduce to

$$
\left.\begin{array}{l}
V_{1}=V_{2}, \\
I_{1}=-I_{2}, \\
A_{1}=B_{2}, \\
B_{1}=A_{2} .
\end{array}\right\}
$$

It will be assumed that the $\gamma_{o m}$ are so chosen that joining equations are always of this form.

The following definitions are useful and serve to bring out further significance of the terminal variables $V_{m}, I_{m}$ and $A_{m}, B_{m}$. The quantity

$$
\frac{V_{m}}{I_{m}} \equiv \gamma_{m m}
$$

is called the impedance of the field on the $m^{\text {th }}$ terminal surface. $\gamma_{m m}$ may be further characterized as a looking-in impedance, since, if the real part of $\gamma_{m m}$ is positive, the average power input at the $m^{\text {th }}$ terminal surface is also positive (eq 5.8). The ratio

$$
\frac{B_{m}}{A_{m}} \equiv \rho_{m m}
$$

is of the nature of a reflection factor. (The term reflection coefficient is ordinarily applied only when all $A$ 's other than $A_{m}$ are zero and the transducer itself does not act as a source.) If the absolute value of $\rho_{m m}$ is less than unity, the average power input at the $m^{\text {th }}$ terminal surface is positive (eq 5.9). From eq 5.6, the relation between $\gamma_{m m}$ and $\rho_{m m}$ is given by

$$
\gamma_{m m}=\gamma_{o m} \frac{1+\rho_{m m}}{1-\rho_{m m}}, \rho_{m m}=\frac{\gamma_{m m}-\gamma_{o m}}{\gamma_{m m}+\gamma_{o m}} .
$$

Both quantities, $\gamma_{m m}$ and $\rho_{m m}$, depend not only on the characteristics of the waveguide transducer involved but also upon the excitation and termination at terminal surfaces other than the $m^{\text {th }}$. Definitions of more general quantities of both types

$$
\frac{V_{k}}{I_{m}} \equiv \gamma_{k m}, \quad \frac{B_{k}}{A_{m}} \equiv \rho_{k m}
$$

should also be recorded. The first is a transfer impedance, the second a transmission factor (cf parenthetical remark above), from the $m^{\text {th }}$ to the $k^{\text {th }}$ terminal surface. These quantities are of course no more characteristic of a waveguide transducer than $\gamma_{m m}$ and $\rho_{m m}$ are.

That particular value of $\gamma_{m m}$ that corresponds to a field consisting solely of an incident wave $\left(\rho_{m m}=0\right)$ is called the characteristic impedance of the field in the $m^{\text {th }}$ waveguide. This characteristic impedance has the arbitrary value $\gamma_{o m}$. The most convenient specific value to use is $\gamma_{o m}=1$ (ohm) $(m=1,2,3, \ldots . n)$, irrespective of the of the characteristics of the waveguide with which it is associated. Since this makes $\gamma_{o m}$ disappear in the formulas, however, it is slightly more informative to leave $\gamma_{o m}$ arbitrary, and it will be left arbitrary in the subsequent general arguments. A set of $V$ 's and $I$ 's or a set of $A$ 's and $B$ 's may be said to represent the fields with which they are associated. It will be convenient to designate the two schemes as $V, I$-representations and $A, B$-representations, respectively. A particular representation of either kind is defined by specifying a particular set of characteristic impedances $\gamma_{o m}$.

The quantities $V_{m}, I_{m}, A_{m}, B_{m}$ are not ordinarily regarded as directly measurable (except possibly $V_{m}, I_{m}$ at low frequencies), although their values in a given case in any chosen representation can be calculated from experimental data if desired. But the actual values of the terminal variables themsevles are seldom of interest. The terminal variables serve as extremely useful auxiliary quantities for the calculation of quantities of more direct interest, such as power, power ratio, impedance etc., which involve only products and ratios of the terminal variables. 
It may be noted that the commonly used values of characteristic impedance, such as, for example,

$$
\left.\begin{array}{c}
\frac{1}{Y} \text { (for all types of waveguide; } \\
\mathrm{Y}=\text { wave admittance) } \\
\frac{h}{w Y} \begin{array}{c}
\text { (for rectangular waveguide } \\
\text { of dimensions } w \times h, h<w)
\end{array} \\
\frac{1}{2 \pi Y} \log \frac{r_{2}}{r_{1}} \text { (for coaxial waveguide, } \\
\text { radii } \left.r_{2}, r_{1} ; r_{2}>r_{1}\right)
\end{array}\right\}
$$

have no special significance in the theory of waveguide transducers. The actual usefulness of the quantities 5.18 as characteristic impedances is largely in the calculation by conventional transmission-line equations of reflection at a plane junction of two waveguides of similar geometry but of differing dimensions or media. ${ }^{9}$ Such calculations, however, are not in the domain of transducer theory.

For principal modes, $V_{m}, I_{m}$ differ in no essential respect from voltage and current as defined in the treatment of transmission lines as circuits with distributed constants. Transmission-line voltage and current may be defined by the line integrals

$$
v_{m}=-\int_{P_{C_{P Q}}}^{Q} \boldsymbol{E}_{m} \cdot d \boldsymbol{s}, \quad i_{m}=\oint_{C_{Q}} \boldsymbol{H}_{m} \cdot d \boldsymbol{s},
$$

where, as shown in figure $3, C_{P Q}$ is any path from a point on conductor $P$ to a point on conductor $Q, C_{Q}$ is any path encircling the conductor $Q$, and both paths of integration are restricted to lie in a transverse surface - the terminal surface $S_{m}$, say. Since the components of curl $\boldsymbol{E}$ and curl $\boldsymbol{H}$ normal to the terminal surface are zero for a principal mode, the line integrals are independent of the particular paths $C_{P Q}, C_{Q}$, and the definitions 5.19 then have meaning. The quantities $v_{m}, i_{m}$ are therefore linear measures of $\boldsymbol{E}_{m}, \boldsymbol{H}_{m}$, respectively, just as are $V_{m}, I_{m}$ in eq 5.4. ${ }^{10}$ This is enough to insure that $v_{m}, i_{m}$, and $V_{m}, I_{m}$ have essentially the same physical meaning whenever

\footnotetext{
${ }^{9}$ It happens that the expressions so obtained are valid if the discontinuity is in the medium only. If a discontinuity in dimensions is involved, only the second and third expressions in 5.18 are applicable, and they yield only a partial or approximate result.

${ }^{10}$ Linear measures of $\boldsymbol{E}_{m}, \boldsymbol{H}_{m}$ for modes other than principal modes may be constructed by means of line integrals. It is then necessary to specify the paths in detail, and the product $\frac{1}{2} V_{m} I_{m}^{*}$ of variables so constructed is pro? portional to power (rather than automatically equal to power).
}

eq 5.19 do have meaning. Numerical agreement between $v_{m}, i_{m}$ and $V_{m}, I_{m}$ can be obtained by suitable choice of $\gamma_{o m}$.

A calculation for the special case of coaxial waveguide will illustrate the above statements. If the radii are $r_{2}, r_{1}\left(r_{2}>r_{1}\right)$, the field-function $\boldsymbol{F}_{o}$ for the principal mode is $\boldsymbol{F}_{o}=N \operatorname{grad}(\log r)$, where $r_{1}<r<r_{2}$, and the normalizing factor $\mathrm{N}=$ $\left(2 \pi \log \frac{r_{2}}{r_{1}}\right)^{-1 / 2}$ makes $\boldsymbol{F}_{o}$ satisfy eq 5.3. Using eq 5.2 , and writing out eq 5.4, one obtains

$$
\left.\begin{array}{l}
\boldsymbol{E}=V N\left(\gamma_{0} Y\right)^{-1 / 2} \operatorname{grad}(\log r), \\
\boldsymbol{H}=I N\left(\gamma_{0} Y\right)^{+1 / 2} \boldsymbol{k} \times \operatorname{grad}(\log r),
\end{array}\right\}
$$

It is expedient to choose $\gamma_{0}=(2 \pi Y)^{-1} \log \frac{r_{2}}{r_{1}}$. If now voltage and current as given by eq 5.19 are calculated from the field given by eq 5.20, one finds

$$
\begin{aligned}
& v=V N\left(\gamma_{o} Y\right)^{-1 / 2} \int_{r_{\mathrm{t}}}^{r_{2}} \operatorname{grad}(\log r) \cdot d \boldsymbol{s}=V, \\
& i=I N\left(\gamma_{o} Y\right)^{1 / 2} \oint k \times \operatorname{grad}(\log r) \cdot d \boldsymbol{s}=I,
\end{aligned}
$$

where the sense of the integration for $i$ corresponds to the positive sense of $\boldsymbol{k}$. Thus the identity of the two definitions is established in a particular case.

The power equation (5.8) and the joining equation (5.12) for waveguide transducers are formally the same as the corresponding equations for ac circuits, in which conventional voltage and current appear as terminal variables. It is instructive to examine, even though briefly, the role of current and voltage from the present point of view. Voltage and current associated with any pair of conductors $P, Q$ may be defined by the line integrals

$$
v_{P Q}=-\int_{P_{C_{P Q}}^{Q}}^{\boldsymbol{E}} \cdot d \boldsymbol{s}, \quad i_{Q}=\oint_{C_{Q}} \boldsymbol{H} \cdot d \boldsymbol{s},
$$

where $\mathrm{C}_{P Q}$ is any path from a point on conductor $P$ to a point on conductor $Q$, and $C_{Q}$ is any path encircling the conductor $Q$, not also encircling conductor $P .{ }^{11}$ These definitions may be applied, for example, to the conductor geometry illustrated in figure 4 , as well as to that of figure 3 , which may now be taken as a particular cross section of a pair of conductors that are not necessarily cylindrical. The definitions 5.21 can have

\footnotetext{
${ }^{11}$ It will be assumed that $i_{P}$, defined by an integral similar to that for $i_{Q}$, is equal and opposite to $i_{Q}$.
} 


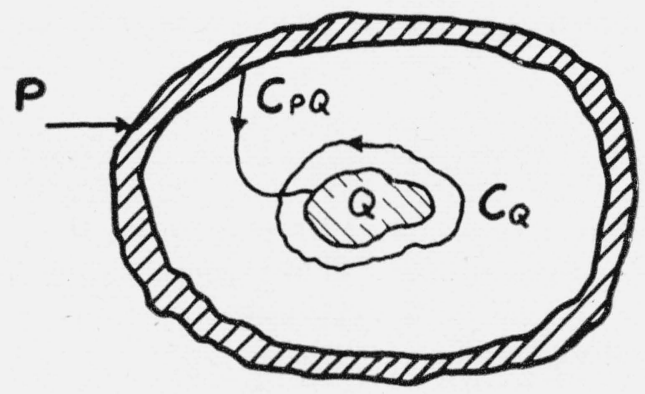

Figure 3. Paths of integration for $v_{m}, i_{m}$.

exact meaning for wholly arbitrary paths $C_{P Q}, C_{Q}$ only for static fields, for which Maxwell's equations imply

$$
\boldsymbol{E}=-\operatorname{grad} \varphi \text {, at all points, }
$$

curl $\boldsymbol{H} \equiv 0$, at all points outside conductors.

Inside conductors, curl $\boldsymbol{H}=\boldsymbol{J}$ (and therefore div $\boldsymbol{J} \equiv 0$ ), where $\boldsymbol{J}$ is the density of conduction current. The concepts of voltage and current, however, are applied not only to static (dc) problems but also to certain nonstatic (ac) problems.

A necessary condition for the applicability of circuit theory, as such, to alternating-current problems is that the dimensions of the system be small enough, and that the frequency be low enough so that, roughly, $\omega \sqrt{\mu \epsilon} d \ll 1$, where $d$ is a representative linear dimension of the apparatus. When this condition is fulfilled, retardation may be neglected, and the field is said to be quasi-stationary. The assumption of a quasi-stationary field does not imply that curl $\boldsymbol{E}$ (everywhere) and curl $\boldsymbol{H}$ (outside conductors) are negligibly different from zero: an inductor is characterized by a nonnegligible value of $j \omega \mu \boldsymbol{H}$, and a capacitor is characterized by a nonnegligible value of $j \omega \mu \boldsymbol{E}$. Thus in the strictest sense of eq 5.21, voltage does not exist in an alternating-current problem if inductance is present, and current does not exist if capacitance is present. Circuit problems are characterized by the fact that regions
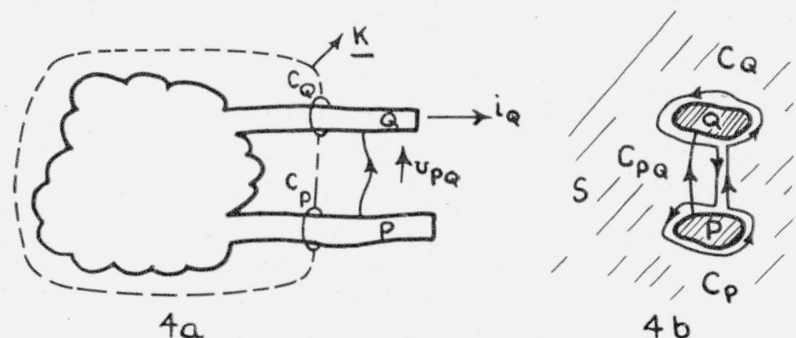

Figure 4. Paths of integration for $v_{P Q}, i_{Q}$. in which the conditions 5.22 seriously fail are localized, and can be isolated by means of suitably drawn terminal surfaces on and near which eq 5.22 may be assumed to be satisfied. For present purposes this may be taken as the qualitative definition of a circuit problem; it leads directly to the existence of voltage and current as terminal variables.

To see more fully what the foregoing statements mean, consider a transducer having just one pair of perfectly conducting wire leads, as illustrated in figure 4,a. The power output from the transducer is

$$
W=\frac{1}{2} \int_{\sigma} \boldsymbol{E} \times \boldsymbol{H}^{*} \cdot \boldsymbol{k} d \sigma,
$$

where $\sigma$ is a surface enclosing the transducer, and $\boldsymbol{k}$ is the outward normal unit vector on $\sigma$. The output power is delivered to whatever system may be outside $\sigma$. Since $\boldsymbol{E} \equiv 0$ in the conductors, the areas cut out of $\sigma$ by the conductors may be omitted from the surface integration. Let $S$ denote the part of $\sigma$ remaining. $S$ is bounded by the two curves $C_{P}$ and $C_{Q}$, and may be made simply connected (if desired) by a cut joining $C_{P}$ and $C_{Q}$ (fig. 4,b). If it is now assumed that the conditions 5.22 hold on $S$, the expression for power may be transformed as follows. The vector identity

$$
\operatorname{curl}\left(\phi \boldsymbol{H}^{*}\right)=(\operatorname{grad} \phi) \times \boldsymbol{H}^{*}+\phi \operatorname{curl} \boldsymbol{H}^{*}
$$

reduces to

$$
\operatorname{curl}\left(\phi \boldsymbol{H}^{*}\right)=-\boldsymbol{E} \times \boldsymbol{H}, *
$$

at points on $S$. Hence, employing Stokes' theorem (with due regard to signs),

$$
\begin{aligned}
W= & -\frac{1}{2} \int_{S} \operatorname{curl}\left(\phi \boldsymbol{H}^{*}\right) \cdot \boldsymbol{k} d S=\frac{1}{2} \oint_{C_{P}} \phi \boldsymbol{H}^{*} \cdot d \boldsymbol{s}+ \\
& \frac{1}{2} \oint_{C \theta} \phi \boldsymbol{H}^{*} \cdot d \boldsymbol{s} .
\end{aligned}
$$

With the choice of signs indicated in figure 4 , and for paths lying in $S$,

$$
\begin{gathered}
v=-\int_{P}^{Q} \boldsymbol{E} \cdot d \boldsymbol{s}=\phi_{Q}-\phi_{P}, \\
i=\oint_{C_{Q}} \boldsymbol{H} \cdot d \boldsymbol{s}=-\oint_{C_{P}} \boldsymbol{H} \cdot d \boldsymbol{s},
\end{gathered}
$$

and the last expression for power becomes

$$
W=\frac{1}{2} v i^{*},
$$


as expected. It may be noted that it was not necessary to make assumptions directly concerning either the region inside $S$ or the region outside $S$. If now $S$ is regarded as a common terminal surface of two transducers (No. 1 inside, No. 2 outside, say), the joining equation for voltage must be

$$
v_{1}=v_{2},
$$

since the surfaces of conductors are equipotential surfaces of a potential function in the neighborhood of a terminal surface. In equating $v_{1}$ and $v_{2}$, the positive senses of the two quantities are assumed to be the same. If the positive directions of $i_{1}$ and $i_{2}$ are directed into the respective transducers at the positive terminal, the joining equations for current are

$$
-i_{1}=i_{2}
$$

since $\operatorname{div} \boldsymbol{J} \equiv 0$ in the neighborhood of a terminal surface, so that current must be continuous.

Voltage and current may be said to represent the terminal fields in a $v, i$-representation (cf p. 522). No arbitrary constants appear in a $v, i$-representation (because none was inserted in the definition of $v$ or of $i$ ). An $a, b$-representation may be formally defined by

$$
\left.\begin{array}{l}
2 a_{m}=v_{m}+\gamma_{c m} i_{m}, \\
2 b_{m}=v_{m}-\gamma_{c m} i_{m} .
\end{array}\right\}
$$

Although these equations are formally the same as the corresponding eq 5.6, the constants $\gamma_{c m}$ which may here be usefully employed are suitably chosen characteristic impedances of the transducers of which $v_{m}, i_{m}$ are terminal variables, rather than characteristic impedances of leads. In fact, quantities with the same physical meaning as the $\gamma_{o m}$ previously used do not exist in the circuit picture. The field is quasi-stationary, and the resolution into incident and emergent waves implied by eq 5.25 is purely formal. But the procedure is useful in network theory as applied to both circuit and wareguide problems; when applied to waveguide transducers it leads to a formal resolution into incident and emergent waves, each of which in general is a linear combination of the physically defined incident and emergent waves.

For completeness, joining equations in an $a, b$-representation should be written down. If, as is assumed, $\gamma_{c 1}=\gamma_{c 2}$, the formal analog of eq 5.14 is

$$
\left.\begin{array}{l}
a_{2}=b_{1}, \\
b_{2}=a_{1} .
\end{array}\right\}
$$

These, of course, follow directly from the definitions 5.25 and 5.24. The generalization of eq 5.26 with $\gamma_{c 1} \neq \gamma_{c 2}$ is not of the same form as the generalization of eq 5.14 with $\gamma_{o 1} \neq \gamma_{o 2}$.

\section{Transducer Theory}

The power equation (5.8) and the joining equations (5.12),

$$
\begin{aligned}
W_{m}=\frac{1}{2} V_{m} I_{m}^{*}, \quad V_{1} & =V_{2}, \\
I_{1} & =-I_{2},
\end{aligned}
$$

provide a basis for the development of transducer theory for the class of waveguide transducers considered. These equations are formally the same as the corresponding equations for circuits. Quite apart from the inherently similar physical meaning of the quantities entering the equations in the waveguide and in the circuit case, the formal identity guarantees that transducer theory developed for the one case is also valid for the other. Those parts of network theory that are truly transducer theory are in fact immediately available for waveguide transducers. Thus, the letter of the purpose implied by the title of this paper was virtually accomplished when eq 5.8 and 5.12 were set up. The purpose of this section is to indicate more fully the methods and the meaning of transducer theory, mostly by presenting selected examples of the theory.

It will be convenient to use the term $2 n$-pole to denote any transducer whose terminal phenomena are harmonically varying electromagnetic fields which can be specified in terms of $n$ pairs of variables $V_{m}, I_{m}$ such that eq 5.8 and 5.12 apply. The variables $V_{m}, I_{m}$ may be of the kind defined for either waveguide or circuit transducers; variables of both kinds may indeed appear in one set of variables for a given transducer. By the above definition, a circuit or a network with $n$ pairs of conventional terminals (or wire leads) is a $2 n$-pole. A waveguide transducer which has $n$ leads and which satisfies a one-mode hypothesis (whether or not in the form specified in sec. IV) is a $2 n$-pole. 
A waveguide transducer which has $n$ waveguide leads and which satisfies a $\lambda_{m}$-mode generalization of the one-mode hypothesis is a $2 \sum_{m=1}^{n} \lambda_{m}$-pole, where $\lambda_{m}$ is the number of nonattenuated modes admitted in the $m^{\text {th }}$ waveguide (appendix). A transducer that has both waveguide leads and wire leads may also be a $2 n$-pole.

Although the term $2 n$-pole is used here in a fairly general sense, the limitation to transducers with electromagnetic terminal phenomena is irrelevant so far as transducer theory is concerned. $V_{m}$ could be interpreted as the amplitude of a harmonically varying force applied at a point of an oscillatory mechanical system, and $I_{m}$ could be interpreted as a corresponding velocity-amplitude. With suitable conventions, the power equations and the joining equations could be set up in the form of eq 5.8 and 5.12, and transducer theory for such systems would then not differ from the theory for $2 n$-poles. These remarks partly suggest the possible generality of transducer theory. Transducer theory can be very general because it only describes or relates external phenomena; it does not attempt to explain or interpret.

A number of topics in the transducer-theory of $2 n$-poles are to be treated in the following paragraphs. In order to be able to proceed, it is necessary to make a basic assumption concerning the number of independent equations that make up the set of equations for a $2 n$-pole (of eq 1.1): It is assumed that the number of such equations is equal to one-half the number of variables in every case. This provides a working rule for the number of equations, but it does not define any particular class of $2 n$-poles. For the purposes of transducer theory a class of $2 n$-poles is defined by specifying the mathematical form of the transducer equations. In the present instance, consideration will be given only to $2 n$-poles that are linear in accordance with the following general definition: A transducer is said to be a linear transducer if the equations of the transducer are linear equations. Additional specializing conditions (for example, reciprocity, losslessness) will be considered in the course of the following discussion. The usefulness of the wealth of mathematical relations that transducer theory can give (of which only a few are given here) is obviously dependent upon the existence of $2 n$-poles which satisfy the various assumed conditions.
Needless to say, the hypotheses mentioned above and others to be employed are not unrealistic.

\section{Linear $2 n$-poles with terminal variables $V_{m}, I_{m}$}

The equations of a $2 n$-pole in terms of terminal variables $V_{m}, I_{m}$ may be written

$$
f_{k}\left(V_{1}, V_{2}, \ldots, V_{n}, I_{1}, I_{2}, \ldots, I_{n}\right) \equiv 0,
$$

where, in accordance with the basic assumption, there are $n$ functions $f_{k}(k=1,2, \ldots, n)$. The $2 n$-poles to be considered are linear; hence, the functions $f_{k}$ may be written

$$
\begin{gathered}
c_{k 1} V_{1}+\ldots+c_{k n} V_{n}+d_{k 1} I_{1}+\ldots+ \\
d_{k n} V_{n}+g_{k} \equiv 0,
\end{gathered}
$$

where $k=1,2, \ldots, n$, and the $c_{k m}, d_{k m}, g_{k}$ are constants independent of $V_{m}, I_{m} \cdot{ }^{12}$ Since there are $n$ independent equations, at least one of the $(2 n) ! /$ $(n !)^{2} n$-rowed determinants of the $n$-by- $2 n$ matrix of the coefficients of the $V_{m}$ and the $I_{m}$ must be different from zero. This means that the system of equations 6.1 can be solved for at least one set of the $(2 n) ! /(n !)^{2}$ different sets of $n$ variables that can be chosen out of the $2 n$ variables $V_{1}$, ..., $V_{n}, I_{1}, \ldots, I_{n}$. This much is known from the general hypotheses. For the purpose of discussion it is assumed that eq 6.1 can, in particular, be solved for the set of $V$ 's or for the set of $I$ 's. ${ }^{13}$ The determinants of the corresponding matrices

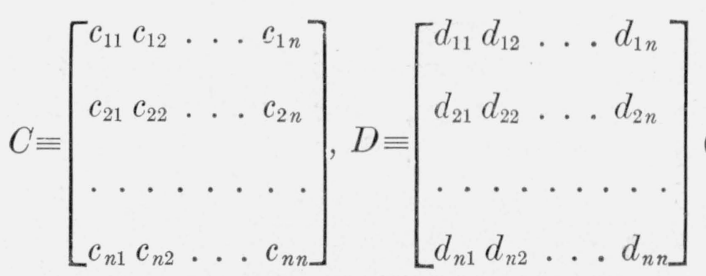

must accordingly be nonzero. With the aid of the matrices $C, D$, and the one-column matrices

$$
V \equiv\left[\begin{array}{c}
V_{1} \\
V_{2} \\
\cdot \\
\cdot \\
\cdot \\
V_{n}
\end{array}\right], \quad I \equiv\left[\begin{array}{c}
I_{1} \\
I_{2} \\
\cdot \\
\cdot \\
I_{n}
\end{array}\right], \quad G \equiv\left[\begin{array}{c}
g_{1} \\
g_{2} \\
\cdot \\
\cdot \\
\cdot \\
g_{h}
\end{array}\right],
$$

12 Equations of the form of eq 6.1, representing the constant and the linear terms of a Taylor's expansion of a general $f_{k}$, can serve as a basis for the firstorder theory of a nonlinear $2 n$-pole.

${ }_{13}$ This assumption is usually, but not always, satisfied in practice. This point comes up again; see p. 536, sec. VII. 
the system of equations (eq. 6.1) may be rewritten as the matrix equation

$$
C V+D I+G \equiv 0 .
$$

To solve for $V$, this equation is multiplied through from the left by $C^{-1}$, the inverse of $C$ :

$$
V=Z I+V_{g},
$$

where $Z \equiv-C^{-1} D, V_{g} \equiv-C^{-1} G$. Similarily, by multiplying eq. 6.3 through by $D^{-1}$,

$$
I=Y V+I_{\mathrm{s}},
$$

where $Y \equiv-D^{-1} C, I_{g} \equiv-D^{-1} G$. The matrix identity $\left(D^{-1} C^{-1}\right)=C^{-1} D$ may be used to obtain the relations

$$
Z=Y^{-1}, \quad V_{g}=-Z I_{g}\left(\text { or } I_{g}=-Y V_{g}\right) .
$$

The significance of the matrices of constants, $Z, Y, V_{g}, I_{g}$, is readily elicited. If the magnetic fields are reduced to zero at all terminal surfaces $(I=0)$, then, from eq $6.3 \mathrm{a}, V=V_{\mathrm{g}}$. Hence the one-column matrix $V_{g}$ is the matrix of opencircuit ${ }^{14}$ electric-field amplitudes: If the electric fields are reduced to zero at all terminal surfaces $(V=0)$, then from eq $6.3 \mathrm{~b}, I=I_{g}$. Hence, the one-column matrix $I_{g}$ is the matrix of the shortcircuit magnetic-field amplitudes. The relation between $V_{g}$ and $I_{g}$, given explicitly in eq 6.4, shows that $I_{g}=0$ if $V_{g}=0$, and conversely. If $V_{g}$ and $I_{g}$ are zero, the $2 n$-pole is said to be sourcefree. ${ }^{15}$

The matrices $Z, Y$ are called the impedance and the admittance matrices, respectively, of the $2 n$ pole. If, for simplicity, the $2 n$-pole is assumed source-free, and if all the elements of $I$ except $I_{p}$ are zero,

$$
V_{q}=Z_{q p} I_{p}
$$

where $Z_{q p}$ is the element in the $q^{\text {th }}$ row and $p^{\text {th }}$ column of $Z$. Thus the value of $Z_{q p}$ is that value of the impedance $\gamma_{q p}=V_{q} / I_{p}$ (cf eq 5.17) which is obtained under the conditions just stated. The elements $Z_{q p}$ are accordingly called open-circuit transfer $(p \neq q)$ or input $(p=q)$ impedances. It is easily verified that the elements $Y_{q p}$ of $Y$ are

\footnotetext{
${ }^{14} \mathrm{It}$ is natural and convenient to adopt certain terms from circuit theory. The use of the terms voltage and current for general $V$ 's and $I$ 's, which may or may not be ordinary voltages and currents, is, however, avoided in this paper. ${ }_{15}$ This is not the same as passive in the standard meaning of the term; the term source-free is here used merely to denote that a $2 n$-pole is such that its equations are homogeneous.
}

appropriately called short-circuit transfer or input admittances. The algebraic relation between $Z$ and $Y$ is given in eq 6.4 .

The most general linear $2 n$-pole can be completely characterized by $n(n+1)$ constants (which are in general complex). In eq 6.3a, for example, there are the $n^{2}$ elements of $Z$, plus the $n$ elements of $V_{\mathrm{g}}$. The original equations (eq 6.1) contain $2 n^{2}+n$ constants, but the nature of the relations connecting the $V$ 's and $I$ 's is determined by $n(n+1)$ combinations of the original constants.

\section{Linear $2 n$-poles with terminal variables $\mathrm{A}_{m}, \mathrm{~B}_{m}$}

Terminal variables $A_{m}, B_{m}$ may be defined in terms of $V_{m}, I_{m}$ by means of the matrix equations

$$
\left.\begin{array}{rl}
V=A+B, & 2 A=V+\gamma_{0} I, \\
\gamma_{0} I=A-B, & 2 B=V-\gamma_{0} I .
\end{array}\right\}
$$

In these equations, $V$ and $I$ are as defined in eq 6.2 ; $A$ and $B$ are one-column matrices with elements $A_{m}$ and $B_{m}(m=1,2, \cdots, n)$, respectively. $\gamma_{o}$ is the diagonal matrix

$$
\gamma_{o}=\left[\begin{array}{lllll}
\gamma_{o 1} & 0 & \cdots & \cdots & 0 \\
0 & \gamma_{o 2} & \cdots & 0 & 0 \\
\ldots & \ldots & \cdots & \ldots \\
0 & 0 & \cdots & \gamma_{o n}
\end{array}\right]
$$

The discussion immediately following, as well as others in which wave amplitudes $A_{m}, B_{m}$ are explicitly involved, will be understood to refer to waveguide transducers. Further, the diagonal elements of $\gamma_{o}$ are precisely the $\gamma_{o m}$ appearing in the definitions of $V_{m}, I_{m}$ (eq 5.4), as indeed the notation indicates. The resolution into waveamplitudes given by the right-hand pair of eq 6.6 is then what may be called physical rather than formal, and the complication of a superposed formal resolution into wave-amplitudes (mentioned on p. 524) will not come into the picture.

Instead of using the relations connecting $A, B$ with $V, I$ to convert the results of section VI, 1 , it is instructive to start afresh with the new variables, and to reapply the reasoning of the previous paragraph. Thus, the equations of a 
$2 n$-pole in terms of terminal variables $A_{m}, B_{m}$ may be written

$$
f_{k}^{\prime}\left(B_{1}, B_{2}, \cdots, B_{n}, A_{1}, A_{2}, \cdots, A_{n}\right) \equiv 0,
$$

where, in accordance with the basic assumption, $k=1,2, \cdots, n$. The $2 n$-poles to be considered are to be linear with respect to $A_{m}, B_{m}$; hence, the functions $f_{k}^{\prime}$ may be written

$c_{k 1}^{\prime} B_{1}+\cdots+c_{k n}^{\prime} B_{n}+d_{k 1}^{\prime} A_{1}+\cdots+d_{k n}^{\prime} A_{n}+g_{k}^{\prime} \equiv 0$,

where $k=1,2, \cdots n$, and the $c_{k m}^{\prime}, d_{k m}^{\prime}, g_{k}^{\prime}$ are constants independent of $B_{m}, A_{m}$. Continuing exactly as with eq 6.1 previously, one is led to the mathematical analogs of eq $6.3 \mathrm{a}, 6.3 \mathrm{~b}$, and 6.4, viz.,

$$
\begin{gathered}
B=S A+B_{g}, \\
A=R B+A_{g}, \\
S=R^{-1}, \quad B_{g}=-S A_{g}\left(\text { or } A_{g}=-R B_{g}\right),
\end{gathered}
$$

where it is assumed that the matrices $R$ and $S$ are both nonsingular.

It is clear that in the absence of incident waves $(A=0)$, the emergent-wave amplitudes are given by $B=B_{g}$; if the incident amplitudes are adjusted so that $A=A_{g}$, the outgoing waves are thereby reduced to zero $(B=0)$. If the $2 n$-pole is such that $A_{g}\left(=B_{g}\right)=0$, it is said to be source-free.

The matrix $S$ is very aptly called the scattering matrix of the $2 n$-pole. If, for simplicity, the $2 n$-pole is assumed source-free, and if all the elements of $A$ except $A_{p}$ are reduced to zero, then the emergent-wave amplitude $B_{q}$ is

$$
B_{q}=S_{q p} A_{p}
$$

where $S_{q p}$ is the element in the $q^{\text {th }}$ row anc the $p^{\text {th }}$ column of $S$. Thus the value of $S_{q p}$ is that value of $\rho_{q p}=B_{q} / A_{p}$ (cf eq 5.17) that is obtained under the conditions just stated. The elements $S_{q p}$ of $S$ are called scattering coefficients. The $S_{q q}$ are also called reflection coefficients; the $S_{p q}(q \neq p)$ are sometimes called transmission coefficients. Mathematically, the interpretation of $R$ is, of course, similar to that of $S$. The matrix $R$ has received little explicit use, and no special terminology for $R$ and its elements is suggested here.

\section{Relationship between Z- and S-matrices}

Since $A, B$ are related to $V, I$ by homogeneous linear equations, it follows that a $2 n$-pole that is linear and source-free in an $A, B$-representation is also linear and source-free in a $V, I$-representation (and vice versa). Quantitative relations between the results of the preceding two paragraphs are easily found. For example, the elimination of $V$ and $I$ from the equation

$$
V=Z I+V_{g}
$$

for a given $2 n$-pole by means of eq 6.6-gives

$$
A+B=Z \gamma_{0}^{-1}(A-B)+V_{g} .
$$

Upon solving this last equation for $B$, and comparing with the equation

$$
B=S A+B_{g}
$$

for the same $2 n$-pole, one obtains

$$
\begin{gathered}
S=\left(Z \gamma_{0}^{-1}+1\right)^{-1}\left(Z \gamma_{0}^{-1}-1\right), \\
B_{g}=\left(Z \gamma_{0}^{-1}+1\right)^{-1} V_{g} .
\end{gathered}
$$

It is easily verified that the two factors in parentheses on the right of eq 6.11 a commute, so that

$$
S=\left(Z \gamma_{0}^{-1}-1\right)\left(Z \gamma_{0}^{-1}+1\right)^{-1} .
$$

For computations, the form

$$
S=1-2\left(Z \gamma_{0}^{-1}+1\right)^{-1}
$$

is convenient. Eq 6.11a solved for Z yields

$$
Z=(1-S)^{-1}(1+S) \gamma_{o}
$$

There are, of course, corresponding relations connecting other pairs of the four matrices $Z, Y$ and $S, R$.

\section{Change of Representation}

The values of the parameters which describe the characteristics of a given linear waveguide transducer are obviously affected by the choice of the $\gamma_{o m}$ in the definitions of the terminal variables (eq 5.4, 5.5). Thus, for example, the transformation of the impedance matrix from a representation defined by $\gamma_{o m}(m=1,2 \cdots n)$ to a representation defined by $\bar{\gamma}_{o m}(m=1,2, \cdots, n)$ may be found as follows. For convenience let $\eta$, $\bar{\eta}$ respectively denote the diagonal matrices whose 
diagonal elements are $\sqrt{\gamma_{o m}}, \sqrt{\gamma_{o m}}$, and let $V, I$ and $\bar{V}, \bar{I}$ denote the corresponding sets of terminal variables. In order that $V, I$ and $\bar{V}, \bar{I}$ represent the same fields, it is necessary that

$$
\left.\begin{array}{c}
\bar{\eta}^{-1} \bar{V}=\eta^{-1} V, \\
\bar{I}=\eta I
\end{array}\right\}
$$

(from eq 5.4). If $\bar{Z}$ and $Z$ are the impedance matrices of a given source-free waveguide $2 n$-pole in the two representations,

$$
\bar{V}=\bar{Z} \bar{I}, \quad V=Z I .
$$

Eliminating $V, I$ from the second of eq 6.14 by means of eq 6.13 , solving for $\bar{V}$,

$$
\bar{V}=\left(\bar{\eta} \eta^{-1} Z_{\eta}^{-1} \bar{\eta}\right) \bar{I},
$$

and comparing with the first of eq 6.14 , one finds

$$
\bar{Z}=\bar{\eta} \eta^{-1} Z_{\eta^{-1}} \bar{\eta}
$$

which is the desired formula. If in particular $\bar{\eta}=1$ (characteristic impedances $\bar{\gamma}_{\text {om }}=1$ ), eq 6.15 normalizes $Z$, and the resulting $\bar{Z}$ is the normalized impedance matrix $z$ of the $2 n$-pole:

$$
z=\eta^{-1} Z \eta^{-1} \text {. }
$$

In terms of the elements of the matrices involved, eq $6.16 \mathrm{a}$ is

$$
z_{k m}=Z_{k m}\left(\gamma_{o k} \gamma_{o m}\right)^{-1 / 2}
$$

A similar type of calculation yields for the scattering matrices $\bar{S}$ and $S$,

$$
\bar{S}=\eta^{-1} \bar{\eta} S \bar{\eta}^{-1} \eta
$$

The normalized scattering matrix is thus

$$
s=\eta^{-1} S \eta,
$$

or, in terms of elements,

$$
s_{k m}=S_{k m}\left(\gamma_{o m} / \gamma_{o k}\right)^{1 / 2} .
$$

It may be noted that the transformation 6.15 does not affect the symmetry properties of an impedance matrix. If an impedance matrix is symmetric in one $\gamma_{o}$-representation, it is symmetric in any $\gamma_{o}$-representation. On the other hand, the transformation 6.17 does affect symmetry properties. A scattering matrix may be symmetric in one representation and unsymmetric in another. This difference in the behavior of impedance matrices and scattering matrices with respect to change of representation can be regarded as a consequence merely of the particular way in which the basic definitions (eq 5.4 and 5.5) were set up.

If the impedance matrix of a $2 n$-pole is symmetric, then the normalized scattering matrix is also symmetric. This may be shown by using the relation $6.11 \mathrm{a}$, which, for the normalized matrices $s$ and $z$, becomes

$$
s=(z+1)^{-1}(z-1) .
$$

Using the tilde to denote the transpose of a matrix,

$$
\begin{aligned}
\tilde{s} & =\widetilde{(z+1)^{-1}(z-1)}=\widetilde{(z-1)} \widetilde{(z+1)^{-1}} \\
& =(\tilde{z}-1)(\widetilde{z}+1)^{-1}=(z-1)(z+1)^{-1}=s .
\end{aligned}
$$

Thus $\tilde{s}=s$, and the symmetry is proved.

\section{Linear two-poles; joining}

For a two-pole, all matrices involved in the foregoing reduce to single numbers. Eq $6.3 \mathrm{a}$ and $6.3 \mathrm{~b}$ reduce to

$$
\begin{aligned}
& V_{1}=Z_{11} I_{1}+V_{g 1}, \\
& I_{1}=Y_{11} V_{1}+I_{g 1},
\end{aligned}
$$

respectively. If $Z_{11}=0$, then eq $6.19 \mathrm{~b}$ has no meaning; if $Y_{11}=0$, then eq $6.19 \mathrm{a}$ has no meaning. $Z_{11}$ and $Y_{11}$ cannot both be zero, on account of the assumed existence of one relation of the form 6.1. If both $Y_{11}$ and $Z_{11}$ are finite, both eq $6.19 \mathrm{a}$ and $6.19 \mathrm{~b}$ apply and they have the same meaning. Moreover,

$$
Y_{11}=Z_{11}^{-1}, \quad V_{g 1}=-Z_{11} I_{g 1}
$$

as in eq 6.4. The content of Thévenin's theorem and of Norton's theorem is included in the meaning of eq 6.19 .

Consider the connection of a two-pole source (whose equation is 6.19a) with a source-free twopole whose equation is $V_{1}^{\prime}=Z_{11}^{\prime} I_{1}^{\prime}$. The system of equations to be solved is then

$$
\left.\begin{array}{rl}
V_{1} & =Z_{11} I_{1}+V_{g 1}, \\
V_{1}^{\prime} & =Z_{11}^{\prime} I_{1}^{\prime}, \\
V_{1}^{\prime} & =V_{1}, \\
I_{1}^{\prime} & =-I_{1},
\end{array}\right\}
$$

Journal of Research 
where the last two equations are the joining equations. Since the two two-poles when connected form a closed system, the number of equations should be sufficient to determine a unique solution for the $V$ 's and $I$ 's. The fact that there are four equations to determine the four variables is a consequence of the basic assumption concerning the number of independent equations for a $2 n$-pole. If $Z_{11}+Z_{11}^{\prime} \neq 0$, the solution of eq 6.20 for $V_{1}^{\prime}, I_{1}^{\prime}$ is obviously

$$
V_{1}^{\prime}=\frac{V_{g 1} Z_{11}^{\prime}}{Z_{11}+Z_{1 \mathrm{I}}^{\prime}}, I_{1}^{\prime}=\frac{V_{\mathrm{g} 1}}{Z_{11}+Z_{11}^{\prime}} .
$$

The appropriate specialization of eq $6.8 \mathrm{a}, 6.8 \mathrm{~b}$ yields the equations of a linear two-pole in an $A, B$-representation:

$$
\begin{aligned}
& B_{1}=S_{11} A_{1}+B_{g 1}, \\
& A_{1}=R_{11} B_{1}+A_{g 1}
\end{aligned}
$$

Remarks similar to those following eq 6.19 apply here also. The calculations with matrices that led to eq 6.11 may be performed for two-poles with numbers. The results are, of course, as in eq 6.11,

$$
\left.\begin{array}{rl}
B_{g 1} & =\frac{V_{g 1}}{Z_{11} \gamma_{o 1}{ }^{-1}+1}, \\
S_{11} & =\frac{Z_{11} \gamma_{o 1}{ }^{-1}-1}{Z_{11} \gamma_{o 1}{ }^{-1}+1}, \\
Z_{11} & =\frac{1+S_{11}}{1-S_{11}} \gamma_{o 1} .
\end{array}\right\}
$$

Consider the connection of a two-pole source (whose equation is 6.21a) with a source-free two-pole whose equation is $B_{1}^{\prime}=S_{11}^{\prime} A_{1}^{\prime}$. The system of equations is then

$$
\left.\begin{array}{l}
B_{1}=S_{11} A_{1}+B_{g 1}, \\
B_{1}^{\prime}=S_{11}^{\prime} A_{1}^{\prime}, \\
B_{1}^{\prime}=A_{1}, \\
A_{1}^{\prime}=B_{1} .
\end{array}\right\}
$$

If $S_{11} S_{11}^{\prime} \neq 1$, the solution of these equations for $B_{1}^{\prime}, A_{1}^{\prime}$ is

$$
B_{1}^{\prime}=\frac{B_{g 1} S_{11}^{\prime}}{1-S_{11} S_{11}^{\prime}}, \quad A_{1}^{\prime}=\frac{B_{g 1}}{1-S_{11} S_{11}^{\prime}} .
$$

\section{Reciprocity}

Suppose that the equations of a $2 n$-pole can be expressed in the form

$$
V_{k}=f_{k}\left(I_{1}, I_{2}, \cdots, I_{n}\right) \quad(k=1,2, \cdots, n) .
$$

Let

$$
\begin{aligned}
& V_{k}^{\prime}=f_{k}\left(I_{1}^{\prime}, I_{2}^{\prime}, \cdots, I_{n}^{\prime}\right) \quad(k=1,2, \cdots, n), \\
& V_{k}^{\prime \prime}=f_{k}\left(I_{1}^{\prime \prime}, I_{2}^{\prime \prime}, \cdots, I_{n}^{\prime \prime}\right) \quad(k=1,2, \cdots, n),
\end{aligned}
$$

where $I_{1}^{\prime}, I_{2}^{\prime}, \cdots, I_{n}^{\prime}$ is one set of independent variables and $I_{1}^{\prime \prime}, I_{2}^{\prime \prime}, \cdots, I_{n}^{\prime \prime}$ is another set of independent variables. Consider the condition

$$
\sum_{m=1}^{n}\left(V_{m}^{\prime} I_{m}^{\prime \prime}-V_{m}^{\prime \prime} I_{m}^{\prime}\right) \equiv 0 ;
$$

this is the reciprocity condition, and it serves to define an important class of $2 n$-poles. The reciprocity condition greatly limits the generality of eq 6.25. To find the form of these equations, first differentiate eq 6.26 with respect to $I_{k}^{\prime \prime}$ obtaining

$$
V_{k}^{\prime}=\sum_{m=1}^{n} \frac{\partial V_{m}^{\prime \prime}}{\partial I_{k}^{\prime \prime}} I_{m}^{\prime}
$$

But $V_{k}^{\prime}$ does not depend upon $I_{1}^{\prime \prime}, I_{2}^{\prime \prime}, \cdots, I_{n}^{\prime \prime}$, so that the differential coefficients in eq 6.27 must be constants independent of $I_{1}^{\prime \prime}, I_{2}^{\prime \prime}, \cdots, I_{n}^{\prime \prime}$. These constants may be denoted by $z_{k m}$, say, and eq 6.27 then states

$$
V_{k}^{\prime}=f_{k}\left(I_{1}^{\prime}, I_{2}^{\prime}, \cdots, I_{n}^{\prime}\right)=\sum_{m=1}^{n} z_{k n} I_{m}^{\prime} ;
$$

that is, the functions $f_{k}$ are linear and homogeneous. Let $V, I$ respectively denote the onecolumn matrices with elements $V_{m}, I_{m}$, and let $Z$ denote the square matrix with elements $z_{k m}$. In this notation eq $6.25 \mathrm{a}$ and eq $6.25 \mathrm{~b}$ become

$$
\begin{gathered}
V^{\prime}=z I^{\prime}, \\
V^{\prime \prime}=z I^{\prime \prime},
\end{gathered}
$$

and eq 6.26 may be rewritten as

$$
\tilde{I}^{\prime \prime} V^{\prime}-\tilde{I}^{\prime} V^{\prime \prime} \equiv 0
$$

where $(\tilde{I})$ denotes the matrix transposed. Eliminating $V^{\prime}, V^{\prime \prime}$ from the last equation gives

$$
\tilde{I}^{\prime \prime} z I^{\prime}-\tilde{I}^{\prime} z I^{\prime \prime} \equiv 0 \text {. }
$$

But $\left(\tilde{I}^{\prime \prime} z I^{\prime}\right)$ is a number, or a matrix of one element, and is therefore symmetric; hence,

$$
\tilde{I}^{\prime \prime} z I^{\prime}=\widetilde{\left(\tilde{I}^{\prime \prime} z I^{\prime}\right)}=\tilde{I}^{\prime} \tilde{z} I^{\prime \prime} .
$$


Eq 6.28 can thus be written

$$
\tilde{I}^{\prime}(\tilde{z}-z) I^{\prime \prime} \equiv 0 .
$$

Since $I^{\prime}$ and $I^{\prime \prime}$, are arbitrary,

$$
\tilde{z}-z=0
$$

the impedance matrix is symmetric. Thus the equations of a $2 n$-pole that satisfies the reciprocity condition are linear and homogeneous and can be written in the form

$$
V=z I
$$

where $z$ is symmetric. The converse is easily verified: if eq 6.29 holds, then the reciprocity condition 6.26 holds. Eq 6.29 is therefore equivalent to eq 6.26 .

If $z$ is symmetric, its inverse, $y=z^{-1}$, is also symmetric. It was shown on p. 528, that if $z$ is symmetric, then the normalized scattering matrix is also symmetric.

\section{Lossless $2 n$-poles}

A $2 n$-pole is said to be lossless if the total timeaverage power input is zero for all values of the terminal variables. Mathematically expressed in terms of $V, I$, this condition is

$$
\operatorname{Re}\left(I^{\dagger} V\right) \equiv 0,
$$

where $I^{\dagger}$ is the transposed complex-conjugate of $I$, and $\operatorname{Re}\left(I^{\dagger} V\right)$ is the real part of $I^{\dagger} V$. If a lossless $2 n$-pole satisfies the reciprocity condition, the following conclusions concerning the form of the equations of the $2 n$-pole can be drawn. Equation 6.30 directly implies

$$
I^{+} V+\tilde{I} V^{*} \equiv 0
$$

Replacing $V$ by $z I$ (eq 6.29),

$$
I^{\dagger} z I+\tilde{I} z^{*} I^{*} \equiv 0
$$

But $\tilde{I}_{\mathrm{z}} * I^{*}$ is a single number and is therefore equal to its transpose $I^{\dagger} z^{\dagger} I$. The last equation becomes

$$
I^{\dagger}\left(z+z^{\dagger}\right) I \equiv 0 .
$$

Since $I$ is arbitrary,

$$
z=-z^{\dagger}
$$

This equation states that the imaginary part of $z$ is symmetric and that the real part is antisym- metric. This much holds for any linear, lossless $2 n$-pole. But since reciprocity was assumed, $z$ is symmetric, and its real part must vanish. Therefore,

$$
z=j x
$$

where $x$ is real and symmetric; $z$ is pure imaginary.

The corresponding properties of the normalized scattering matrix may be derived from eq 6.32 via eq $6.11 \mathrm{a}$, or directly from the counterpart of eq 6.30 in an $A, B$-representation. Choosing the latter method, the needed power equation is, in matrix notation,

$$
A^{\dagger} A-B^{\dagger} B \equiv 0
$$

(eq 5.9 with $\gamma_{o m}=1$ ). Inserting $B=s A$,

$$
A^{\dagger} A-A^{\dagger} s^{\dagger} s A \equiv 0 .
$$

Since $A$ is arbitrary,

$$
s^{\dagger} s=1 .
$$

$\mathrm{Eq} 6.34$ states that the inverse of $s$ is the transposed complex conjugate of $s$; a matrix having this property is known as a unitary matrix. This much holds for any linear, lossless $2 n$-pole. But since $s$ is symmetric, $s^{\dagger}=s^{*}$, and eq 6.34 becomes

$$
s^{*} s=1 ;
$$

the inverse of $s$ is simply the complex conjugate of $s$.

\section{Four-poles}

Four-poles are of particular importance because more complicated transducers are often built up of a number of four-poles connected in tandem. If a four-pole satisfies the reciprocity condition, its equations may be written in either of the two forms

$$
\left.\begin{array}{l|l}
V_{1}=z_{11} I_{1}+z_{12} I_{2}, & B_{1}=s_{11} A_{1}+s_{12} A_{2}, \\
V_{2}=z_{12} I_{1}+z_{22} I_{2}, & B_{2}=s_{12} A_{1}+s_{22} A_{2},
\end{array}\right\}
$$

where the matrices $z$ and $s$ are symmetric, as written. It is often convenient to have these relations expressed in the so-called transmissionline form, defined by

$$
\left.\begin{array}{l|l}
V_{1}=t_{11} \bar{V}_{2}+t_{12} \bar{I}_{2}, & B_{1}=r_{11} \bar{B}_{2}+r_{12} \bar{A}_{2}, \\
I_{1}=t_{21} \bar{V}_{2}+t_{22} \bar{I}_{2}, & A_{1}=r_{21} \bar{B}_{2}+r_{22} \bar{A}_{2},
\end{array}\right\}
$$


where $\bar{V}_{2} \equiv V_{2}, \bar{I}_{2} \equiv-I_{2}$; and where $\bar{B}_{2} \equiv A_{2}, \bar{A}_{2} \equiv B_{2}$. The reason for making the definition with respect to the quantities distinguished by bars will become apparent. The new coefficients are given in terms of the coefficients in eq 6.36 by

$$
\left.\begin{array}{lr}
t_{11}=z_{11} / z_{12}, & t_{12}=\left(z_{11} z_{22}-z_{12}^{2}\right) / z_{12}, \\
t_{21}=1 / z_{12}, & t_{22}=z_{22} / z_{12} . \\
r_{11}=-\left(s_{11} s_{22}-s_{12}^{2}\right) / s_{12}, & r_{12}=s_{11} / s_{12}, \\
r_{21}=-s_{22} / s_{12}, & r_{22}=1 / s_{12} .
\end{array}\right\}
$$

It is important to note that reciprocity is now manifested in the relations

$$
t_{11} t_{22}-t_{12} t_{21}=1 ; \quad \mid \quad r_{11} r_{22}-r_{12} r_{21}=1 ;
$$

i. e., the determinant of the matrices of coefficients in eq 6.37 has the value unity. One may easily verify that this is both a necessary and a sufficient condition that the matrices of coefficients in the original eq 6.36 be symmetric.

If a two-pole whose equations are

$$
\bar{V}_{2}=z_{\gamma} \bar{I}_{2} \quad \mid \quad \bar{B}_{2}=s_{\tau} \bar{A}_{2}
$$

is connected to the above four-pole at terminal surface No. 2, then eq 6.37 yield

$$
\frac{V_{1}}{I_{1}}=\gamma_{11}=\frac{t_{11} z_{\tau}+t_{12}}{t_{21} z_{\tau}+t_{22}} . \quad \frac{B_{1}}{A_{1}}=\rho_{11}=\frac{r_{11} s_{r}+r_{12}}{r_{21} s_{\tau}+r_{22}} .
$$

Thus the input impedance $\gamma_{11}$ (or reflection coefficient $\rho_{11}$ ) is exhibited as a linear fractional function of the load impedance $z_{\tau}$ (or reflection factor $s_{r}$ ). Transformations of the form 6.41 have many interesting and useful mathematical properties. (Equations of this form hold for any linear source-free four-pole, whether or not the reciprocity condition is satisfied).

Suppose that a second four-pole is given, whose equations in the transmission-line form are

$$
\begin{array}{l|l}
V_{1}^{\prime}=t_{11}^{\prime} \bar{V}_{2}^{\prime}+t_{12}^{\prime} \bar{I}_{2}^{\prime}, & B_{1}^{\prime}=r_{11}^{\prime} \bar{B}_{2}^{\prime}+r_{12}^{\prime} \bar{A}_{2}^{\prime}, \\
I_{1}^{\prime}=t_{21}^{\prime} \bar{V}_{2}^{\prime}+t_{22}^{\prime} \bar{I}_{2}^{\prime}, & A_{1}^{\prime}=r_{21}^{\prime} \bar{B}_{2}^{\prime}+r_{22}^{\prime} \bar{A}_{2}^{\prime},
\end{array}
$$

where the notation is in all respects similar to that of eq 6.37. Let it be required to find the characteristics of the four-pole formed by joining the two given four-poles, say with terminal surface No. 2 of the first coinciding with terminal surface No. 1 of the second. The notation has been chosen so that the corresponding mathematical process is merely a linear substitution. The result, in matrix notation, is

$\left[\begin{array}{l}V_{1} \\ I_{1}\end{array}\right]=\left(T T^{\prime}\right)\left[\begin{array}{l}\overline{V_{2}^{\prime}} \\ \bar{I}_{2}^{\prime}\end{array}\right], \quad \mid\left[\begin{array}{l}B_{1} \\ A_{1}\end{array}\right]=\left(R R^{\prime}\right)\left[\begin{array}{c}\overline{B_{2}^{\prime}} \\ \overline{A_{2}^{\prime}}\end{array}\right]$,

where $T$ and $R$ are the matrices of eq $6.37, T^{\prime}$ and $R^{\prime}$ are the matrices of eq 6.42. Thus the matrix of the parameters of the combination of the two four-poles is the matrix product of the matrices of the separate four-poles taken in the proper order. If the four-pole described by eq 6.42 satisfies the reciprocity condition, the determinants $\left|T^{\prime}\right|$ and $\left|R^{\prime}\right|$ are each equal to unity. In this case, from the general rule for the multiplication of determinants,

$$
\left|T T^{\prime}\right|=1 \quad|\quad| R R^{\prime} \mid=1
$$

and the composite four-pole also satisfies the reciprocity condition. (Reciprocity was assumed for the first four-pole, eq 6.39).

\section{Linear symmetric four-poles}

A four-pole is said to be symmetric if it exhibits the same electrical characteristics when viewed from either of its two terminal surfaces. Thus the normalized $z$ - or $s$-matrix of a linear symmetric (source-free) four-pole must be unaltered by the interchange of subscripts 1 and 2. That is, it is necessary that

$$
z_{11}=z_{22}, \quad z_{12}=z_{21} \cdot \mid s_{11}=s_{22}, \quad s_{12}=s_{21} .
$$

A linear symmetric four-pole therefore satisfies the reciprocity condition and the additional condition $z_{11}=z_{22} \quad$ (or $\left.s_{11}=s_{22}\right)$. If a four-pole is lossless, as well as linear, symmetric, and source-free, then $z$ must be pure imaginary and $s$ must be unitary. The equations of a four-pole satisfying these special conditions are obtained in a familiar form as follows: $z$ can be written

$$
z=\left[\begin{array}{ll}
j x_{11} & j x_{12} \\
j x_{12} & j x_{11}
\end{array}\right]
$$


where $x_{11}, x_{12}$ are real. Defining $x_{11} / x_{12}=a$, $-1 / x_{12}=b$, the coefficients of the transmissionline form of the equations of the four-pole become (eq 6.38)

$$
\begin{array}{ll}
t_{11}=a, & t_{12}=j\left(1-a^{2}\right) / b, \\
t_{21}=j b, & t_{22}=a .
\end{array}
$$

A characteristic impedance $\gamma_{c}$ of a four-pole is one that is transformed into itself by the transformation 6.41. For the four-pole in question, $\gamma_{c}$ is given by

$$
\gamma_{c}=\frac{a \gamma_{c}+j\left(1-a^{2}\right) / b}{j b \gamma_{c}+a}
$$

which reduces to $b^{2} \gamma_{c}^{2}+a^{2}=1$. Since the constants $b \gamma_{c}$ and $a$ satisfy $b^{2} \gamma_{c}^{2}+a^{2}=1$, they can be written

$$
\begin{aligned}
a & =\cos \theta, \\
b \gamma_{c} & =\sin \theta,
\end{aligned}
$$

as a mere matter of notation. $\quad \gamma_{c}$ is real if $|a|<1$; $\theta$ is then also real. $\gamma_{c}$ is pure imaginary if $|a|>1$; $\theta$ is then also pure imaginary. The four-pole may be characterized in terms of $\theta, \gamma_{c}$ by eliminating $a, b$ from the expressions for $t_{11}, t_{12}$, etc. The resulting matrix is

$$
T=\left[\begin{array}{ll}
\cos \theta & j \gamma_{c} \sin \theta \\
j \frac{1}{\gamma_{c}} \sin \theta & \cos \theta
\end{array}\right] .
$$

For any fixed value of $\gamma_{c}$, the matrix is defined by the value of $\theta, T=T(\theta)$. If four-poles represented by $T\left(\theta_{1}\right)$ and $T\left(\theta_{2}\right)$ are joined, the matrix of the composite four-pole is $T\left(\theta_{1}\right) T \theta_{2}$ ). Upon writing out the matrix product it is found that

$$
T\left(\theta_{1}\right) T\left(\theta_{2}\right)=T\left(\theta_{1}+\theta_{2}\right) .
$$

Equation 6.45 shows that $T(0)=1$; eq 6.46 thus yields

$$
T(\theta) T(-\theta)=1,
$$

or, $T^{-1}(\theta)=T(-\theta) . \quad$ It is apparent from eq 6.46 that the matrices $T\left(\theta_{1}\right), T\left(\theta_{2}\right)$ commute with each other.

\section{Translation of terminal surfaces}

As a further and final specialization, consider four-poles of the type represented by eq 6.45 with $\gamma_{c}=1$ prescribed. The corresponding $T$-matrix is

$$
T(\theta)=\left[\begin{array}{lr}
\cos \theta & j \sin \theta \\
j \sin \theta & \cos \theta
\end{array}\right] .
$$

The $R$-matrix (eq 6.37) corresponding to this $T$-matrix is particularly simple. From the definitions 6.6 (with $\gamma_{o m}=1$, since a normalized representation was assumed in section VI, 9) and from the definitions following eq 6.37 one finds:

$$
\begin{aligned}
{\left[\begin{array}{l}
V_{1} \\
I_{1}
\end{array}\right] } & =\left[\begin{array}{c}
B_{1}+A_{1} \\
-B_{1}+A_{1}
\end{array}\right]=\Omega\left[\begin{array}{l}
B_{1} \\
A_{1}
\end{array}\right],\left[\begin{array}{l}
\bar{V}_{2} \\
\bar{I}_{2}
\end{array}\right]=\left[\begin{array}{c}
\bar{B}_{2}+\bar{A}_{2} \\
-\bar{B}_{2}+\bar{A}_{2}
\end{array}\right] \\
& =\Omega\left[\begin{array}{l}
\bar{B}_{2} \\
\bar{A}_{2}
\end{array}\right],
\end{aligned}
$$

where

$$
\Omega \equiv\left[\begin{array}{rr}
1 & 1 \\
-1 & 1
\end{array}\right] .
$$

The relations connecting $V_{1}, I_{1}$ and $\bar{V}_{2}, \bar{I}_{2}$ are

$$
\left[\begin{array}{c}
V_{1} \\
I_{1}
\end{array}\right]=T(\theta)\left[\begin{array}{l}
\bar{V}_{2} \\
\bar{I}_{2}
\end{array}\right] .
$$

Using eq 6.48 to eliminate the $V$ 's and $I$ 's,

$$
\Omega\left[\begin{array}{l}
B_{1} \\
A_{1}
\end{array}\right]=T(\theta) \Omega\left[\begin{array}{l}
\bar{B}_{2} \\
\bar{A}_{2}
\end{array}\right] .
$$

from which one obtains

$$
\left[\begin{array}{l}
B_{1} \\
A_{1}
\end{array}\right]=\Omega^{-1} T(\theta) \Omega\left[\begin{array}{l}
\bar{B}_{2} \\
\bar{A}_{2}
\end{array}\right] .
$$

Therefore the matrix $R(\theta)$ relating $B_{1}, A_{1}$ and $\bar{B}_{2}, \bar{A}_{2}$ is

$$
R(\theta)=\Omega^{-1} T(\theta) \Omega .
$$

For the particular $T(\theta)$ considered, $R(\theta)$ is readily found to be

$$
R(\theta)=\left[\begin{array}{ll}
e^{-j \theta} & 0 \\
0 & e^{j \theta}
\end{array}\right] .
$$

$R(\theta)$ obviously shares the properties 6.46 of $T(\theta)$. The transformation of the reflection factor (eq 6.41 ) defined by $R(\theta)$ is very simple, viz.,

$$
\rho_{11}=e^{-2 j \theta} s_{r} .
$$


Among the four-poles that can be characterized by matrices of the form of $R(\theta)$ (or $T(\theta)$ ) are included all those consisting of a section of ideal waveguide. This is seen by employing eq 6.49 to write

$$
\left.\begin{array}{l}
B_{1}=\bar{B}_{2} e^{-j \theta}+0, \\
A_{1}=0 \quad+\bar{A}_{2} e^{j \theta},
\end{array}\right\}
$$

and comparing these equations with eq 5.1. It is evident that $\theta$ is to be identified as $\beta L$, where $L$ is the length of the section of ideal waveguide considered, and $\beta$ is the appropriate phase constant. $(R(\theta)$ and $T(\theta)$ were set up for $\theta$ real, corresponding to the real values of $\beta$ for any nonattenuated mode.). The determination of $\beta$ for a given mode in a given waveguide is a matter of experìment or of electromagnetic theory; $\beta$ cannot be determined in transducer theory. It is thus logical to continue to use the electrical length $\theta$, in preference to writing $\beta L$ for the same quantity.

It was remarked on p. 517 that a translation of a terminal surface of a waveguide transducer is equivalent to joining a transducer consisting of a section of ideal waveguide at the terminal surface in question. The corresponding mathematical process is given by eq 6.43 for four-poles. Thus, if a four-pole has a matrix $T$ defined with respect to given terminal surfaces, and if terminal surface No. 1 is shifted an electrical distance $\theta_{1}$, the corresponding new matrix is given by

$$
T^{\prime}=T\left(\theta_{1}\right) T
$$

If, also, terminal surface No. 2 is shifted by the electrical distance $\theta_{2}$, the result is

$$
T^{\prime \prime}=T^{\prime} T\left(\theta_{2}\right)=T\left(\theta_{1}\right) T T\left(\theta_{2}\right)
$$

Consider next the joining of any two given fourpoles with matrices $T_{1}, T_{2}$, where the matrices are

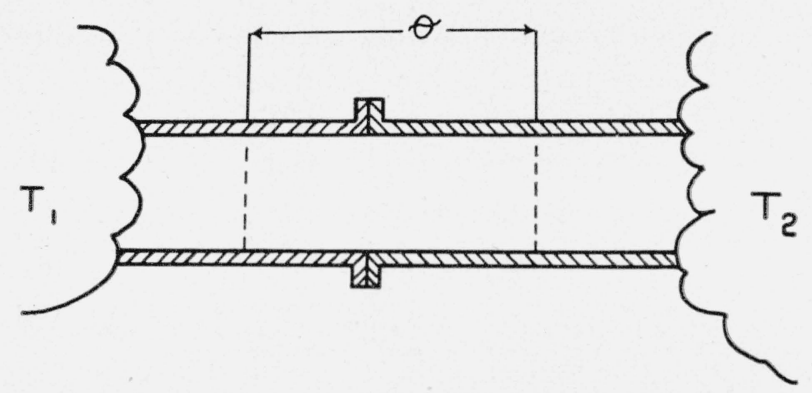

FigURE 5. Junction of two four-poles. defined with respect to terminal surfaces which do not necessarily coincide when the physical junction is made (fig. 5).

The matrix of the physically defined composite four-pole is then given mathematically as a composite of three four-poles,

$$
T=T_{1} T(\theta) T_{2},
$$

where $\theta$ is the electrical distance between the terminal surfaces involved ( $\theta$ may be positive or negative).

The results $6.52,6.53$ when expressed in terms of $R$-matrices, are, of course, of exactly the same form as eq 6.52 and eq 6.53 themselves. The implied algebraic details, while simple for the $T$-matrices, are exceedingly simple for the $R$ matrices. The simplicity of many calculations for four-poles in an $A, B$-representation in fact recommends the use of this representation of the terminal fields in many problems. For example, the transformation of the characteristics of a waveguide $2 n$-pole corresponding to translation of the $n$ terminal surfaces is readily obtained by working directly with the scattering matrix of the $2 n$-pole and applying the basic properties of the $R$-transformation as expressed by eq 6.51.

\section{The reciprocity theorem}

The determination of the characteristics of a given $2 n$-pole is fundamentally a matter of experiment. If the details of the structure of a $2 n$-pole are given, it is sometimes possible to replace direct experimental measurement by theoretical calculations based more or less directly upon Maxwell's equations. A circuit problem is usually regarded as specified in detail when the component capacitances, inductances, etc., and their interconnections are given. With such data given, the calculation of the characteristics of a $2 n$-pole is set up in accordance with Kirchhoff's laws for electric circuits, and the calculation is a relatively simple algebraic problem. If, however, instead of capacitances, inductances, etc., the geometry and the electrical constants of the media making up the capacitors, inductors, etc., are given, the problem in general becomes more difficult, and the solution must be based more directly on the field equations. Microwave problems are characterized by the specification of geometry and electrical constants and by the necessity of em- 
ploying the field equations. The solution of microwave problems in detail is in general extremely difficult. Much of the difficulty arises because of the vector nature of the field, and because retardation cannot be neglected.

The difficulty of detailed calculation of the behavior of microwave components emphasizes the importance of transducer theory in calculations and measurements in microwave work. Conditions of the type employed in section VI lead at once to certain necessary characteristics of the equations of any $2 n$-pole satisfying the various conditions. Moreover, the applicability of one or more of those conditions (such as linearity, reciprocity, losslessness, symmetry) is in many cases evident without explicit experimental verification. Reciprocity, in particular, is a very general and very important property and is perhaps the least obvious of those mentioned. The purpose of this section is to give a proof of a rather general reciprocity theorem for $2 n$-poles.

The reciprocity theorem is to be proved for a class of $2 n$-poles satisfying the following condition: The media involved must be such that Maxwell's equations become linear equations at all interior points of the $2 n$-pole. ${ }^{16}$ No restriction is imposed upon the geometry of the structure admitted, other than that indirectly imposed by the requirement that the structure shall actually be a $2 n$-pole. The geometry may, for example, be that of coils, capacitors, and wires or that of cavity resonators and waveguides. It should be noted that the hypothesis of linearity here employed is of a very different type from that employed in section VI. The important difference is that here the hypothesis applies directly to all points of the interior of a $2 n$-pole, whereas there it applied only to externally observable behavior at the terminals. Reciprocity for a $2 n$-pole composed of circuit elements is usually regarded as a consequence of Kirchhoff's laws. Under the assumption of passive, linear, bilateral circuit-elements, Kirchhoff's laws yield the reciprocity theorem almost at once. The basic assumption employed in this section (viz., linear media) differs very much in form and slightly in content, from the circuit-hypotheses just mentioned. Under the present hypothesis of linearity, Maxwell's equations are employed to deduce a reciprocity

\footnotetext{
${ }^{16} \mathrm{~A}$ more complete statement of the hypotheses is given below.
}

theorem holding for any kind of $2 n$-pole satisfying the hypothesis. The theorem will therefore apply to waveguide transducers, circuits, and to the mixed type involving both circuit and waveguide leads. Some further preliminaries are needed in preparation for the proof of the theorem.

It is necessary to define precisely what is meant by the interior of a $2 n$-pole. The region occupied by the electromagnetic field of a $2 n$-pole is denoted by $R$, and the boundary of $R$ is denoted by $S$. $R$ and $S$ are illustrated for a waveguide transducer with two waveguide leads in figure 6 . If the

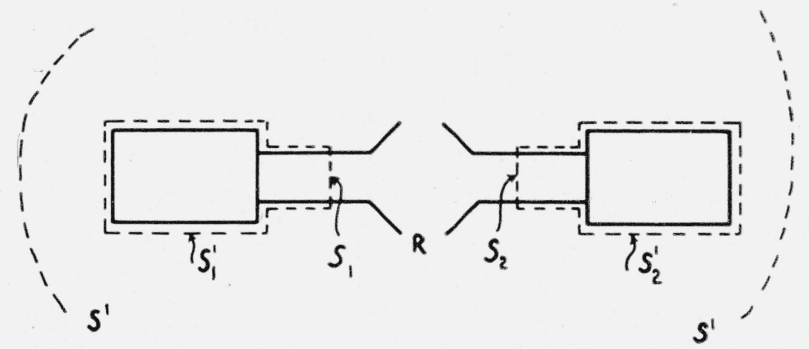

Figure 6. Surface $S$ for a waveguide transducer.

number of leads is $n$, the surface $S$ may be thought of as consisting of $n+1$ separate parts, viz., a large spherical surface $S^{\prime}$ (with center at some mean position in the region), plus $n$ separate closed surfaces $S_{m}^{\prime}(m=1,2, \ldots, n)$. The surface $S_{m}^{\prime}$ extends transversely a cross the $m^{\text {th }}$ waveguide (coinciding with the terminal surface $S_{m}$ within the waveguide), and encloses the two-pole source terminating the $m^{\text {th }}$ lead. The region $R$, the interior of the $2 n$-pole, is thus bounded externally by the surface $S^{\prime}$ and internally by the surfaces $S_{m}^{\prime}$. The surfaces $S_{m}^{\prime}$ enclose and thereby exclude the fields and currents belonging to the two-pole sources.

A $2 n$-pole of the type suggested in figure 6 is by no means completely shielded; its field extends to infinity. The radius of the spherical surface $S^{\prime}$ must be allowed to become infinite for any incompletely shielded $2 n$-pole. Furthermore, in order that all of the field of such a $2 n$-pole be included in $R$, the surfaces $S_{m}^{\prime}$ must fit closely over the surfaces of the two-pole sources, and these must be assumed to be perfectly shielded. A region $R$ and surface $S$ of the type just described serve for any waveguide transducer. If a waveguide transducer is completely shielded, as illustrated in figure 7, an alternative $R$ and $S$ may be drawn as shown in the figure. The subsequent argument will employ the notation of the preceding figure 6 , 


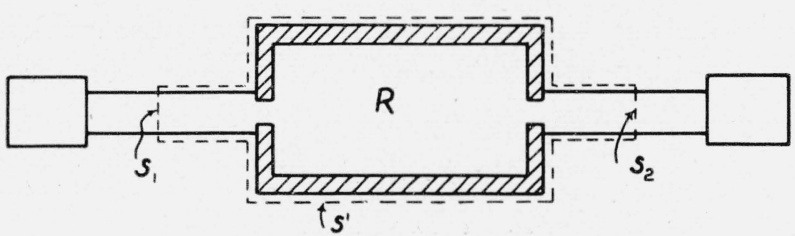

FIG URE 7. Surface $S$ for an enclosed waveguide transducer.

but no essential changes are required to adapt the argument to the simpler but less general case llustrated in figure 7 .

The following proof will also apply to $2 n$-poles having one or more circuit terminals of the type discussed in section $\mathrm{V}$ and illustrated in figure 4 . Thus any or all of the two-pole sources of the type illustrated in figure 6 could be replaced by those of the type illustrated in figure 4 . If this replacement is made for the $m^{\text {th }}$ lead, say, the surface $S_{m}^{\prime}$ (of fig. 6) becomes the terminal surface of the $m^{\text {th }}$ two-pole source. No important changes in the argument are involved.

The complete statement of the hypothesis imposed on the media in $R$ is made as follows: Maxwell's equations in a very general form are

$$
\left.\begin{array}{rl}
\operatorname{curl} \boldsymbol{E} & =-\frac{\partial}{\partial t} \boldsymbol{B}(\boldsymbol{H}), \\
\operatorname{curl} \boldsymbol{H} & =+\frac{\partial}{\partial t} \boldsymbol{D}(\boldsymbol{E})+\boldsymbol{J}(\boldsymbol{E}) .
\end{array}\right\}
$$

The vectors $\boldsymbol{B}, \boldsymbol{D}, \boldsymbol{J}$ are respectively the magnetic induction, the electric displacement, and the induced current density. The functions $\boldsymbol{B}(\boldsymbol{H})$, $\boldsymbol{D}(\boldsymbol{E}), \boldsymbol{J}(\boldsymbol{E})$ describe the media involved. It is required that the media be such that these functions are linear and homogeneous. The most general homogeneous linear vector functions may be written

$$
\left.\begin{array}{l}
\boldsymbol{B}(\boldsymbol{H})=\mathscr{l} \cdot \boldsymbol{H} \\
\boldsymbol{D}(\boldsymbol{E})=\mathscr{E} \cdot \boldsymbol{E} \\
\boldsymbol{J}(\boldsymbol{E})=\mathscr{Y} \cdot \boldsymbol{E}
\end{array}\right\}
$$

where the tensors $\mathbb{I}, \mathscr{E}, \mathscr{Y}^{\prime}$, (which may have both real and imaginary parts) represent respectively permeability, dielectric constant, and conductivity. These tensors depend upon the position coordinates; they may depend upon the frequency $\omega$, but not upon $\boldsymbol{E}, \boldsymbol{H}$, and the time. If, as is assumed, all sources of the field vary harmonically with time at frequency $\omega$, the solution of eq 7.1 subject to eq 7.2 may be obtained in the form $\boldsymbol{E}=\boldsymbol{E}_{0} e^{j \omega t}, \boldsymbol{H}=\boldsymbol{H}_{0} e^{j_{\omega} t}$, where $\boldsymbol{E}_{0}, \boldsymbol{H}_{0}$ are functions only of position. Inserting $\boldsymbol{E}, \boldsymbol{H}$ of this form in eq 7.1, the governing equations become

$$
\left.\begin{array}{l}
\operatorname{curl} \boldsymbol{E}=-j \omega \mathscr{I} \cdot \boldsymbol{H}, \\
\operatorname{curl} \boldsymbol{H}=+j \omega \cdot \boldsymbol{E}+\mathscr{Y} \cdot \boldsymbol{E} .
\end{array}\right\}
$$

Equations 7.3 determine the position-dependence of the complex amplitudes of a field which varies harmonically with frequency $\omega$ at every point of $R$. In accordance with the hypotheses introduced in section IV, eq 7.3 must reduce to eq 4.1 in the neighborhood of terminal surfaces.

The tensors $\mathbb{I}, \mathscr{S}, \mathscr{S}$ of course reduce to scalars for isotropic media. If tensors are required to express the properties of the media, it is essential for the proof of reciprocity that the tensors be symmetric tensors. Symmetry of $\mathbb{I}, \sigma$ is sometimes deduced from energy considerations, at least when . // are real. It is here assumed that $/ /, \mathscr{E}, \mathcal{Y}$ are in fact symmetric.

Particular solutions of the eq 7.3 for a given $2 n$-pole are determined by the boundary conditions, which may be expressed in terms of the values of $\boldsymbol{k} \times \boldsymbol{E}$ and $\boldsymbol{k} \times \boldsymbol{H}$ on $S$, where $\boldsymbol{k}$ is the inward unit normal vector on $S$. It is a theorem of electromagnetic theory that the solution of Maxwell's equations in $R$ is uniquely determined for all times $t>0$ by the values of $\boldsymbol{E}, \boldsymbol{H}$ throughout $R$ at $t=0$ and the values of $\boldsymbol{k} \times \boldsymbol{E}$ or $\boldsymbol{k} \times \boldsymbol{H}$ on $S .{ }^{17}$ (The values of $\boldsymbol{k} \times \boldsymbol{E}$ may be specified over part of $S$, and the values of $\boldsymbol{k} \times \mathbf{H}$, over the remaining part of $S$.) If, as is assumed, the $2 n$-pole under consideration is such that power is dissipated (however slightly), the effects of the initial values of the field throughout $R$ will be transient, and the steadystate field (the solution of eq 7.3) will be determined solely by the values of $\boldsymbol{k} \times \boldsymbol{E}$ and $\boldsymbol{k} \times \boldsymbol{H}$ on $S$.

The terminal surfaces $S_{m}$ form a part of $S$. On the terminal surfaces, $\boldsymbol{k} \times \boldsymbol{E}$ and $\boldsymbol{k} \times \boldsymbol{H}$ are given by $\boldsymbol{E}_{m}$ and $\boldsymbol{H}_{m}$ (sec. V). On the remaining parts of the surfaces $S_{m}^{\prime}$ the field satisfies the homogeneous boundary condition $\boldsymbol{k} \times \boldsymbol{E}=0$. On $S^{\prime}$ the field is to satisfy the so-called outward-radiating condition, which insures that there are no sources outside $S^{\prime}$ (i. e., at infinity), and matters only for nonshielded $2 n$-poles. It follows from the uniqueness theorem and from these boundary conditions

17 J A. Stratton, Electromagnetic Theory, p. 486 (MeGraw-Hill Book Co. New York, N. Y., 1941). 
that the solution of eq 7.3 is uniquely determined by a set of $n$ terminal fields such that either $\boldsymbol{k} \times \boldsymbol{E}$ or $\boldsymbol{k} \times \boldsymbol{H}$ is specified on each of the $n$ terminal surfaces. A set of such fields is illustrated in table 1. No condition is placed upon the two-pole sources responsible for the terminal fields, other than that they produce fields of frequency $\omega$. The nature of the ultimate sources that generate the fields is of no importance. So far as the $2 n$-pole under consideration is concerned, it matters only that certain fields do appear at its terminals.

TABLE 1. A set of terminal fields

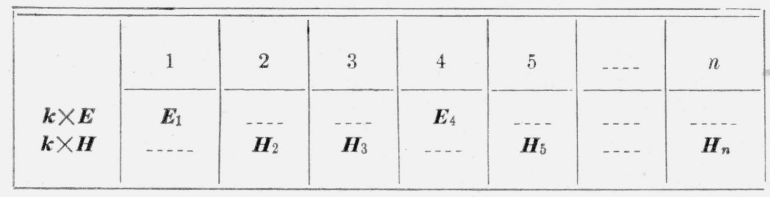

It has been tacitly assumed that a solution exists for arbitrary values of the terminal fields in sets of the type shown in table 1 . The uniqueness theorem insures merely that if a solution exists, it is unique. In order to carry through the proof of the reciprocity theorem, it is assumed that the set $\boldsymbol{H}_{1}, \boldsymbol{H}_{2}, \cdots, \boldsymbol{H}_{n}$ may be arbitrarily prescribed..$^{18}$

The proof of the reciprocity theorem is made to rest on two lemmas. The first of these is a very general reciprocity relation first given by $\mathrm{H}$. A. Lorentz. Let $\boldsymbol{E}^{\prime}, \boldsymbol{H}^{\prime}$ and $\boldsymbol{E}^{\prime \prime}, \boldsymbol{H}^{\prime \prime}$ be two fields arising from independent and arbitrary sets of sources with frequency $\omega$. The fields satisfy eq 7.3 in $R$,

$$
\begin{aligned}
\operatorname{curl} \boldsymbol{E}^{\prime} & =-j \omega \cdot \mathscr{U} \cdot \boldsymbol{H}^{\prime}, \\
\operatorname{curl} \boldsymbol{H}^{\prime} & =+j \omega \mathscr{E} \cdot \boldsymbol{E}^{\prime}+\mathscr{Y} \cdot \boldsymbol{E}^{\prime}, \\
\operatorname{curl} \boldsymbol{E}^{\prime \prime} & =-j \omega \mathscr{U} \cdot \boldsymbol{H}^{\prime \prime}, \\
\operatorname{curl} \boldsymbol{H}^{\prime \prime} & =+j \omega \mathscr{5} \cdot \boldsymbol{E}^{\prime \prime}+\mathscr{Y} \cdot \boldsymbol{E}^{\prime \prime} .
\end{aligned}
$$

Define the vector $\boldsymbol{L}=\boldsymbol{E}^{\prime} \times \boldsymbol{H}^{\prime \prime}-\boldsymbol{E}^{\prime \prime} \times \boldsymbol{H}^{\prime}$, and form the vector identity

$$
\begin{gathered}
\operatorname{div} \boldsymbol{L}=\boldsymbol{H}^{\prime \prime} \cdot \operatorname{curl} \boldsymbol{E}^{\prime}-\boldsymbol{E}^{\prime} \cdot \operatorname{curl} \boldsymbol{H}^{\prime \prime} \\
-\boldsymbol{H}^{\prime} \cdot \operatorname{curl} \boldsymbol{E}^{\prime \prime}+\boldsymbol{E}^{\prime \prime} \cdot \operatorname{curl} \boldsymbol{H}^{\prime} .
\end{gathered}
$$

18 The existence of a solution is of course to be expected in any problem with a genuine physical pedigree. Eut in practice, certain idealizations are useful, and the assumption made in the text is not always valid. For example, if the equations of a four-role are those of an ideal 1:1 transformer,

$$
\begin{aligned}
V_{1} & =V_{2}, \\
-I_{1} & =I_{2},
\end{aligned}
$$

neither the rair ${ }^{-} V_{1}, V_{2}$ nor the pair $I_{1}, I_{2}$ can te chosen arbitrarily. But either of the pairs $V_{1}, I_{1}$ or $V_{2}, I_{2}$ can be chosen arbitrarily, and corresponding solutions for the fields exist and are unique.
Substituting the expressions 7.4 for the curls, and making use of the symmetry of the tensor parameters, one obtains

$$
\operatorname{div} \boldsymbol{L}=0(\text { in } R \text { ), }
$$

which relation is the first lemma.

The second lemma asserts that the integral of the normal component of $\boldsymbol{L}$, taken over the sperical surface $S^{\prime}$, vanishes:

$$
\int_{S^{\prime}} \boldsymbol{n} \cdot\left(\boldsymbol{E}^{\prime} \times \boldsymbol{H}^{\prime \prime}-\boldsymbol{E}^{\prime \prime} \times \boldsymbol{H}^{\prime}\right) d S=0 .
$$

This is most easily shown by considering the asymptotic form of the fields on $S^{\prime}$ for large values of radius $r$ (but the fact to be proved is independent of the size and shape of $S^{\prime}$ ). For sufficiently large $r$, the field approaches that of a concentrated source at $r=0$, viz.,

$$
\begin{aligned}
& \boldsymbol{E}^{\prime}=\boldsymbol{t}^{\prime}{\frac{e^{-j \boldsymbol{\beta} r}}{r}}, \\
& \boldsymbol{H}^{\prime}=Y_{0} \boldsymbol{n} \times \boldsymbol{E}^{\prime}, \\
& \boldsymbol{E}^{\prime \prime}=\boldsymbol{t}^{\prime \prime} \frac{e^{-j \beta_{0} r}}{r}, \\
& \boldsymbol{H}^{\prime \prime}=Y_{0} \boldsymbol{n} \times \boldsymbol{E}^{\prime \prime} \text {, }
\end{aligned}
$$

where $\boldsymbol{t}$ is a transverse vector function $(\boldsymbol{t} \cdot \boldsymbol{n}=0)$ of the direction of the vector $r, \beta_{0}=\omega \sqrt{\mu \epsilon}$, and $Y_{0}=\sqrt{\epsilon / \mu}$. (It is assumed that for large $r$ the medium is simply free space.) The fields described by eq 7.7 are in general elliptically polarized spherical waves. Upon substituting eq 7.7 into eq 7.6 one finds that the integrand of eq 7.6 vanishes identically, since

$$
\boldsymbol{t}^{\prime} \times\left(\boldsymbol{t}^{\prime \prime} \times \boldsymbol{n}\right) \equiv \boldsymbol{t}^{\prime \prime} \times\left(\boldsymbol{t}^{\prime} \times \boldsymbol{n}\right) .
$$

Hence one may infer that

$$
\lim _{r=\infty} \int_{S^{\prime}} \boldsymbol{n} \cdot \boldsymbol{L} d S=0,
$$

which is the statement of the second lemma.

After the foregoing preliminaries, the proof of the reciprocity theorem is very direct. By the divergence theorem,

$$
\int_{R} \operatorname{div} \boldsymbol{L} d \tau=-\int_{S} \boldsymbol{k} \cdot \boldsymbol{L} d S
$$

(The minus sign appears because $k$ was chosen as the inward normal.) By the first lemma, $\operatorname{div} \boldsymbol{L}=0$ in $R$, so that 


$$
\int_{S} \boldsymbol{k} \cdot \boldsymbol{L} d S=0
$$

Hence, by the definition of $S$,

$$
\sum_{m=1}^{n} \int_{S_{m}^{\prime}} \boldsymbol{k} \cdot \boldsymbol{L} d S+\int_{S^{\prime}} \boldsymbol{k} \cdot \boldsymbol{L} d S=0
$$

And by the second lemma, the integral over $S^{\prime}$ vanishes, at least in the limit as $r \rightarrow \infty$. But the terms of the summation in eq 7.9 do not depend upon $r$, so that

$$
\sum_{m=1}^{n} \int_{S_{m}^{\prime}} \boldsymbol{k} \cdot \boldsymbol{L} d S=0
$$

(and the integral over $S^{\prime}$ must in fact be independent of $r$ ). Replacing $\boldsymbol{L}$ by the quantity for which it is an abbreviation,

$$
\sum_{m=1}^{n} \int_{S_{m}^{\prime}} \boldsymbol{k} \cdot\left(\boldsymbol{E}^{\prime} \times \boldsymbol{H}^{\prime \prime}-\boldsymbol{E}^{\prime \prime} \times \boldsymbol{H}^{\prime}\right) d S=0 .
$$

The integral over $S_{m}^{\prime}$ reduces in all cases to the integral over the terminal surface $S_{m}$. (For waveguide terminal surfaces, the integrand vanishes on those parts of $S_{m}^{\prime}$ that do not coincide with $S_{m}$.) Hence

$$
\sum_{m=1}^{n} \int_{S_{m}} \boldsymbol{k}_{m} \cdot\left(\boldsymbol{E}_{m}^{\prime} \times \boldsymbol{H}_{m}^{\prime \prime}-\boldsymbol{E}_{m}^{\prime \prime} \times \boldsymbol{H}_{m}^{\prime}\right) d S=0 .
$$

The individual terms of this expression are of the same form as the power relation 5.8 (or 5.23). Therefore

$$
\sum_{m=1}^{n}\left(V_{m}^{\prime} I_{m}^{\prime \prime}-V_{m}^{\prime \prime} I_{m}^{\prime}\right)=0 .
$$

Since this relation holds for arbitrary values of the independent variables, it is precisely equivalent to the reciprocity condition 6.26. This completes the proof of the reciprocity theorem.

The algebraic consequences of the reciprocity condition were examined in section VI, 6. It was there shown that the equations of a $2 n$-pole satisfying the reciprocity condition are expressible by means of the homogeneous linear equations

$$
V_{k}=\sum_{m=1}^{n} Z_{k m} I_{m}(k=1,2, \cdots, n),
$$

where the matrix of coefficients $Z_{k m}$ is symmetric. The symmetry of the Z-matrix is the essential content of the reciprocity theorem; the linearity and homogeneity of the equations of the $2 n$-pole can be inferred directly from the same properties of Maxwell's equations in $R$.

\section{A reactance theorem}

By an application of Poynting's theorem, one can obtain a fundamental formula relating the impedance matrix of a $2 n$-pole to the fields in the interior of the $2 n$-pole. Consider a $2 n$-pole that satisfies the hypotheses used in section VII, assuming now for simplicity that the media involved are isotropic. Assume also that $\mu$ and $\epsilon$ are real; dielectric losses, if present, are to be represented by an equivalent conductivity included in $\sigma$. Poynting's theorem (for the complex Poynting's vector) applied to the region $R$ bounded by the surface $S$ states that

$$
\begin{aligned}
& \frac{1}{2} \int_{S} \boldsymbol{E} \times \boldsymbol{H}^{*} \cdot \boldsymbol{k} d S=\int_{R} \frac{1}{2} \sigma \boldsymbol{E} \cdot \boldsymbol{E}^{*} d \tau \vdash \\
& j \omega \int_{R}\left(\frac{\mu}{2} \boldsymbol{H} \cdot \boldsymbol{H}^{*}-\frac{\epsilon}{2} \boldsymbol{E} \cdot \boldsymbol{E}^{*}\right) d \tau
\end{aligned}
$$

where the notation is carried over from section VII. As in section VII, the surface integral may be split into the sum of integrals over the terminal surfaces plus an integral over the spherical surface $S^{\prime}$. The sum of the integrals over the terminal surfaces reduces to the matrix product $\frac{1}{2} I^{\dagger} V$, or to $\frac{1}{2} I^{\dagger} Z I$, where $Z I$ replaces $V$. Eq 8.1 becomes

$$
\begin{aligned}
\frac{1}{2} I^{\dagger} Z I= & \frac{1}{2} \int_{S^{\prime}} \boldsymbol{E} \times \boldsymbol{H}^{*} \cdot \boldsymbol{n} d S+\int_{R} \frac{1}{2} \sigma \boldsymbol{E} \cdot \boldsymbol{E}^{*} d \tau+ \\
& j \omega \int_{R}\left(\frac{\mu}{2} \boldsymbol{H} \cdot \boldsymbol{H}^{*}-\frac{\epsilon}{2} \boldsymbol{E} \cdot \boldsymbol{E}^{*}\right) d \tau,
\end{aligned}
$$

where $\boldsymbol{n}$ denotes the outward normal on $S^{\prime}$. For a shielded $2 n$-pole, the $S^{\prime}$-integral contributes nothing, since the integrand vanishes. If the $2 n$-pole is not shielded, the radius $r$ of the surface $S^{\prime}$ must be made to become infinite, and $R$ becomes a region of infinite extent. The contribution of the $S^{\prime}$-integral to the right-hand side of eq 8.2 is then real (as may be seen by reference to the form of the field for large $r$, eq 7.7), and represents the time-average of the power lost by radiation. (For many circuit $2 n$-poles, even if unshielded, the radiation loss is negligible.) The second term on the right of eq 8.2 gives the Joulean and the dielectric power losses in the $2 n$-pole. The last term, which is pure imaginary, is $2 j \omega$ times the dif- 
ference of the time-average magnetic and electric energies of the field of the $2 n$-pole. Writing $Q$ for the total power dissipation, and $U_{H}, U_{E}$ for the magnetic and the electric energies, eq 8.2 becomes for the special case of a two-pole,

$$
Z_{11}=R_{11}+j X_{11}=\frac{2}{\left|I_{1}\right|^{2}}\left[Q+2 j \omega\left(U_{H}-U_{E}\right)\right] .
$$

This equation throws some light on the physical meaning of impedance: $R_{11}$ is determined by the losses; $X_{11}$, by the reactive energy unbalance.

It has already been remarked that the calculation of the field, particularly in microwave problems, is in general very difficult. On the other hand, it is not difficult to derive a general property of the reactance matrix of a lossless $2 n$-pole. The property in question is a generalization of an essential part of the content of Foster's reactance theorem for circuits, viz., the frequency-derivative of the reactance of a lossless linear two-pole is positive,

$$
\frac{d X_{11}}{d \omega}>0
$$

For $2 n$-poles, the corresponding statement is that the frequency-derivative of the reactance matrix is positive definite. A proof of this statement will now be given for the generalized type of $2 n$-poles considered in this paper.

The field of a lossless $2 n$-pole that satisfies the hypotheses of the reciprocity theorem (sec. VII) is subject to Maxwell's equations in the form

$$
\left.\begin{array}{l}
\operatorname{curl} \boldsymbol{E}=-j \omega \mu \boldsymbol{H}, \\
\operatorname{curl} \boldsymbol{H}=+j \omega \epsilon \boldsymbol{E} .
\end{array}\right\}
$$

The parameters $\mu, \epsilon$ must be real; for simplicity it is assumed that $\mu, \epsilon$ are scalars and that they are independent of frequency in the frequency-range considered. (It is sufficient that $\partial \mathscr{E} / \partial \omega$ and

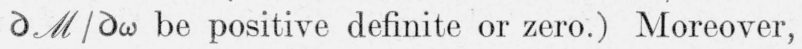
the $2 n$-pole must be completely shielded so that radiation loss cannot occur. The domain of the field $\boldsymbol{E}, \boldsymbol{H}$ is a region $R$ bounded by the surface $S^{\prime \prime}$ with inward normal $\boldsymbol{k}$. Since the physical boundaries of the field must be perfectly conducting, the boundary condition $\boldsymbol{k} \times \boldsymbol{E}=0$ must be satisfied everywhere on $S^{\prime \prime}$ except on the terminal surfaces $S_{m}$ (which form a part of $S^{\prime \prime}$ ). Let the fields on the terminal surfaces be specified in terms of a set of magnetic field amplitudes ("currents" in the circuit case) $I_{1}, I_{2}, \cdots, I_{n}$. A solution of eq $8.4 \mathrm{a}$ is thereby determined, and the corresponding electric field amplitudes $V_{m}$ are related to the $I_{m}$ by the matrix equation

$$
V=Z(\omega) I
$$

where $Z(\omega)$ is the impedance matrix (at frequency $\omega$ ) of the $2 n$-pole, and $V, I$ are one-column matrices with elements $V_{m}$ and $I_{m}$, respectively. Since the reciprocity theorem applies, $Z(\omega)$ is symmetric; and since the $2 n$-pole is by hypothesis lossless, $Z(\omega)$ is pure imaginary (sec. VI, 7). Hence, in place of $V=Z(\omega) I$, one may write

$$
V=j X(\omega) I,
$$

where $X(\omega)$ is real and symmetric.

Let $\boldsymbol{E}^{\prime}, \boldsymbol{H}^{\prime}$ denote the $2 n$-pole field satisfying the boundary conditions determined by $I_{1}, I_{2}, \cdots$, $I_{m}$ at a new frequency $\omega^{\prime}=\omega+\delta \omega$. The field equations corresponding to eq 8.4a are

$$
\left.\begin{array}{l}
\operatorname{curl} \boldsymbol{E}^{\prime}=-j \omega^{\prime} \mu \boldsymbol{H}^{\prime}, \\
\operatorname{curl} \boldsymbol{H}^{\prime}=+j \omega^{\prime} \epsilon \boldsymbol{E}^{\prime},
\end{array}\right\}
$$

and the matrix equation corresponding to eq $8.5 \mathrm{a}$ is

$$
V^{\prime}=j X\left(\omega^{\prime}\right) I .
$$

The matrix $I$ is arbitrary, but by hypothesis it is the same for both eq $8.5 \mathrm{a}$ and eq $8.5 \mathrm{~b}$.

It should be noted that in a waveguide, the terminal fields determined by $I$ are not necessarily the same for the two frequencies $\omega^{\prime}$ and $\omega$. For, going back to eq 5.4, 5.2, one finds that $H_{m}$ and $H_{m}^{\prime}$ are given by

$$
\begin{aligned}
& \boldsymbol{H}_{m}=I_{m}\left(\gamma_{o m} Y_{m}\right)^{1 / 2} \boldsymbol{k}_{m} \times \boldsymbol{F}_{o m}, \\
& \boldsymbol{H}_{m}^{\prime}=I_{m}\left(\gamma_{o m}^{\prime} Y_{m}^{\prime}\right)^{1 / 2} \boldsymbol{k}_{m} \times \boldsymbol{F}_{o m},
\end{aligned}
$$

where the primes denote quantities associated with the frequency $\omega^{\prime}$. The wave admittance $Y_{m}$ depends on frequency for modes other than principal modes; $\boldsymbol{F}_{\text {om }}$, however, is a function of geometry and does not depend upon frequency. The subsequent calculation is appreciably simplified by choosing $\gamma_{o m}(\omega)=\left[Y_{m}(\omega)\right]^{-1}$. The $V$, $I$-representation to be used thus depends upon frequency in a particular way, and this necessarily affects the frequency-dependence of $X(\omega)$. With this choice of representation, the last two equations above are replaced by the single equation 


$$
\boldsymbol{H}_{m}=I_{m} \boldsymbol{k}_{m} \times \boldsymbol{F}_{o m},
$$

which holds for both $\omega$ and $\omega^{\prime}$. The corresponding equations for $\boldsymbol{E}_{m}$ and $\boldsymbol{E}_{m}^{\prime}$ are

$$
\left.\begin{array}{l}
\boldsymbol{E}_{m}=V_{m} \boldsymbol{F}_{o m}, \\
\boldsymbol{E}_{m}^{\prime}=V_{m}^{\prime} \boldsymbol{F}_{o m},
\end{array}\right\}
$$

as obtained from eq. 5.4, 5.2 with $\gamma_{o m}=\frac{1}{Y_{m}}$. The discussion leading to eq 8.6 and 8.7 pertains only to waveguide terminal fields. For terminals of circuit type the question of representation does not arise. The special choice made above permits the calculation to be carried through in one formal manner for any kind of $2 n$-pole - waveguide, circuit, or mixed.

By forming the quantity $\boldsymbol{E}^{\prime} \times \boldsymbol{H}^{*}-\boldsymbol{E} \times \boldsymbol{H}^{\prime *}$, integrating its inward normal component over $S^{\prime \prime}$, and applying the divergence theorem, one obtains

$$
\begin{aligned}
& \frac{1}{2} \sum_{m=1}^{n} \int_{S m}\left(\boldsymbol{E}^{\prime} \times \boldsymbol{H}^{*}-\boldsymbol{E} \times \boldsymbol{H}^{*}\right) \cdot \boldsymbol{k}_{m} d S= \\
& \frac{1}{2} \int_{R} \operatorname{div}\left(\boldsymbol{E} \times \boldsymbol{H}^{\prime *}-\boldsymbol{E}^{\prime} \times \boldsymbol{H}^{*}\right) d \tau,
\end{aligned}
$$

since $\boldsymbol{E}^{\prime} \times \boldsymbol{k}=\boldsymbol{E} \times \boldsymbol{k}=0$ on the conducting surfaces. Using eq. 8.6, 8.7, and the normalizing condition 5.3, the left-hand side of eq. 8.8 is reduced to

$$
\frac{1}{2}\left(I^{\dagger} V^{\prime}-I^{\dagger} V\right)
$$

where $I^{\dagger}$ is the transposed complex-conjugate of I. From eq 8.5, this quantity may be written

$$
\frac{1}{2} j I^{+}\left[X\left(\omega^{\prime}\right)-X(\omega)\right] I
$$

which is, to the first order in $\delta \omega$, equal to

$$
\frac{1}{2} j\left(I^{+} \frac{d X}{d \omega} I\right) \delta \omega
$$

Since $X(\omega)$ is real and symmetric, $d X / d \omega$ is also real and symmetric, and the quantity 8.9 is therefore pure imaginary.

The right-hand side of eq 8.8 is transformed as follows: The integrand is, by a vector identity,

$$
\operatorname{div}\left(\boldsymbol{E} \times \boldsymbol{H}^{\prime *}-\boldsymbol{E}^{\prime} \times \boldsymbol{H}^{*}\right)=\boldsymbol{H}^{*} \cdot \operatorname{curl} \boldsymbol{E}-
$$$$
\boldsymbol{E} \cdot \operatorname{curl} \boldsymbol{H}^{\prime *}-\mathrm{H}^{*} \cdot \operatorname{curl} \boldsymbol{E}^{\prime}+\boldsymbol{E}^{\prime} \cdot \operatorname{curl} \boldsymbol{H}^{*} \text {. }
$$

Maxwell's equations 8.4 serve to eliminate the curls:

$$
\begin{aligned}
& \operatorname{div}\left(\boldsymbol{E} \times \boldsymbol{H}^{\prime *}-\boldsymbol{E}^{\prime} \times \boldsymbol{H}^{*}\right)=-j \omega\left(\mu \boldsymbol{H} \cdot \boldsymbol{H}^{\prime *}+\right. \\
& \left.{ }_{\epsilon} \boldsymbol{E}^{\prime} \cdot \boldsymbol{E}^{*}\right)+j \omega^{\prime}\left(\mu \boldsymbol{H}^{\prime} \cdot \boldsymbol{H}^{*}+\epsilon \boldsymbol{E} \cdot \boldsymbol{E}^{\prime *}\right) .
\end{aligned}
$$

Letting $\boldsymbol{H}^{\prime} \equiv \boldsymbol{H}+\delta \boldsymbol{H}, \quad \boldsymbol{E}^{\prime} \equiv \boldsymbol{E}+\delta \boldsymbol{E}, \omega^{\prime}=\omega+\delta \omega$, and expanding the right-hand side of the last equation, one obtains the quantity

$-2 \omega \operatorname{Imag}\left(\epsilon \boldsymbol{E} \cdot \delta \boldsymbol{E}^{*}+\mu \boldsymbol{H}^{*} \cdot \delta \boldsymbol{H}\right)+j \delta \omega\left(\epsilon \boldsymbol{E} \cdot \boldsymbol{E}^{*}+\mu \boldsymbol{H} \cdot \boldsymbol{H}^{*}\right)$,

correct to the first order. Hence, the right-hand side of eq 8.8 may be written as

$$
\begin{aligned}
& -\omega \operatorname{Imag} \int_{R}\left(\epsilon \boldsymbol{E} \cdot \delta \boldsymbol{E}^{*}+\mu \boldsymbol{H}^{*} \cdot \delta \boldsymbol{H}\right) d \tau+ \\
& j \delta \omega \int_{R}\left(\frac{\epsilon}{2} \boldsymbol{E} \cdot \boldsymbol{E}^{*}+\frac{\mu}{2} \boldsymbol{H} \cdot \boldsymbol{H}^{*}\right) d \tau .
\end{aligned}
$$

The first term of this expression must actually be zero to the first order in $\delta \omega$, since the term is real, and the real part of the left-hand side of eq 8.8 is zero to the first order. This information, however, is incidental; the desired result is obtained from the equality of the imaginary terms of eq 8.8. From eq 8.9 and 8.10, then,

$$
\frac{1}{2} j\left(I^{+} \frac{d X}{d \omega} I\right) \delta \omega=j \delta \omega \int_{R}\left(\frac{\epsilon}{2} \boldsymbol{E} \cdot \boldsymbol{E}^{*}+\frac{\mu}{2} \boldsymbol{H} \cdot \boldsymbol{H}^{*}\right) d \tau .
$$

Therefore

$$
I^{\dagger} \frac{d X}{d \omega} I=4\left(U_{H}+U_{E}\right),
$$

where $U_{H}, U_{E}$ represent magnetic and electric energies, as in eq 8.3. Since the quantity $U_{H}+U_{E}$ is positive for every nonzero $I$, eq 8.11 states that the frequency derivative of the reactance matrix is positive definite, as was to be shown. (For $\omega$ approaching a resonance frequency, $U_{H}+U_{E}$ tends to infinity, so that $d X / d \omega$ is not defined at such exceptional frequencies.)

It is perhaps worthwhile to write down the generalization of eq 8.11 holding for arbitrary choice of the $\gamma_{o m}$. Let an arbitrary representation of the fields at waveguide terminal surfaces be defined by the diagonal matrix $\gamma_{o}$ with diagonal elements $\gamma_{01}, \gamma_{02}, \ldots, \gamma_{o n}$, and let $Y_{0}$ denote the diagonal matrix of wave-admittances with diagonal elements $Y_{1}, Y_{2}, \ldots, Y_{n}$. (If some of the terminals of the $2 n$-pole are circuit terminals, the corresponding $\gamma_{o m}$ and $Y_{m}$ are to be understood to be equal to unity.) Then the reactance matrix $X$ 
associated with the representation $\gamma_{0}$ is such that ${ }^{19}$

$$
I^{\dagger} \gamma_{0} Y_{0} \frac{d}{d \omega}\left(\gamma_{0}^{-1} Y_{0}^{-1} X\right) I=4\left(U_{H}+U_{E}\right)
$$

In a normalized representation, $\gamma_{0}=1$, and eq 8.12 simplifies to

$$
I^{\dagger} Y_{0} \frac{d}{d \omega}\left(Y_{0}^{-1} x\right) I=4\left(U_{H}+U_{E}\right)
$$

where $x$ is the normalized reactance matrix. The equation is of the same form for any set of frequency-indepdendent $\gamma_{o}{ }^{\prime}$ s. If the $\gamma_{o m}$ are chosen so that the product $\gamma_{o} Y_{o}$ is independent of frequency (as was done in the derivation of 8.11 ), eq 8.12 reduces to eq 8.11 , as it should.

\section{Appendix}

\section{Multimode Interaction}

The extension of the discussion of the text to include multimode interaction, as defined in section IV, is straightforward. An outline of this generalization will suffice.

Equations of the form of eq 5.1, 5.2, 5.3 hold for each mode $\eta$ in waveguide $m$. The amplitude coefficients $V_{m}^{\eta}, I_{m}^{\eta}$ of mode $\eta$ in waveguide $m$ are defined by

$$
\left.\begin{array}{rl}
\boldsymbol{E}_{m}^{\eta} & =V_{m}^{\eta} \boldsymbol{E}_{o m}^{\eta}, \\
\boldsymbol{H}_{m}^{\eta} & =I_{m}^{\eta} \boldsymbol{H}_{a m}^{\eta} .
\end{array}\right\}
$$

(A multiplier $\gamma_{o m}^{\eta}$, which could be inserted, as in eq 5.4, is here omitted for simplicity.) The tangential components of $\boldsymbol{E}$ and $\boldsymbol{H}$ on the $m^{t h}$ terminal surface are

$$
\left.\begin{array}{l}
\boldsymbol{E}_{m}=\sum_{\eta=1}^{\lambda_{m}} \boldsymbol{E}_{m}^{\eta}=\sum_{\eta=1}^{\lambda_{m}} V_{m}^{\eta} \boldsymbol{E}_{o m}^{\eta}, \\
\boldsymbol{H}_{m}=\sum_{\eta=1}^{\lambda_{m}} \boldsymbol{H}_{m}^{\eta}=\sum_{\eta=1}^{\lambda_{m}} I_{m}^{\eta} \boldsymbol{H}_{o m}^{\eta},
\end{array}\right\}
$$

where $\lambda_{m}$ denotes the number of nonattenuated modes admitted in waveguide $m$. The complex power input at the $m^{t h}$ terminal surface is given by

$$
W_{m}=\frac{1}{2} \int_{S_{m}}\left(\boldsymbol{E}_{m} \times \boldsymbol{H}_{m}^{*}\right) \cdot \boldsymbol{k}_{m} d S .
$$

Because of the orthogonality property of the waveguide modes, $W_{m}$ can be written as the sum of contributions from the individual modes,

10 The $X$ in eq 8.12 is related to the $X$ in eq 8.11 by a transformation of the type discussed in sec. VI, 4. where

$$
W_{m}=\sum_{\eta=1}^{\lambda_{m}} W_{m}^{\eta}
$$

$$
W_{m}^{\eta}=\frac{1}{2} \int_{S_{m}}\left(\boldsymbol{E}_{m}^{\eta} \times \boldsymbol{H}_{m}^{\eta^{*}}\right) \cdot \boldsymbol{k}_{m} d S=1 / 2 V_{m}^{\eta} I_{m}^{\eta^{*}} .
$$

This is the basic power expression. Turning to the joining equations, and referring to the notation and the discussion on p. 520 , section $\mathrm{V}$, it is clear that

$$
\begin{aligned}
\boldsymbol{E}_{1} & \equiv \boldsymbol{E}_{2}, \\
\boldsymbol{H}_{1} & \equiv \boldsymbol{H}_{2}
\end{aligned}
$$

express the joining condition. From the orthogonality (or from the linear independence) of the waveguide modes, it follows that the amplitudes must satisfy

$$
\left.\begin{array}{c}
V_{1}^{\eta}=V_{2}^{\eta}, \\
I_{1}^{\eta}=-I_{2}^{\eta},
\end{array}\right\}
$$

where $\eta=1,2, \ldots, \lambda_{1}$ (and $\lambda_{1}=\lambda_{2}$, of course).

Equations 5.8', 5.12' are formally identical to the corresponding equations previously obtained. Thus a multimode waveguide transducer can be treated as a $2 M$-pole, where $M=\sum_{m=1}^{n} \lambda_{m}$. The transducer theory of section VI can be adapted to the multimode case without formal change in that theory by the expedient of numbering the variables $V_{m}^{\eta}, I_{m}^{\eta}$ serially in some order and denoting them by $V_{m}, I_{m}$, where $m=1,2, \ldots, M$. With this notation the reciprocity theorem of section VII and the reactance theorem of section VIII apply formally unchanged.

For some purposes, however, it is convenient to retain the superscript identification of mode and the subscript identification of waveguide. The equations of a linear source-free waveguide transducer, having $n=2, \lambda_{1}=2$, $\lambda_{2}=3$, for example, may be written

$$
\left[\begin{array}{l}
V_{1}^{1} \\
V_{1}^{2} \\
V_{2}^{1} \\
V_{2}^{2} \\
V_{2}^{3}
\end{array}\right]=\left[\begin{array}{lllll}
Z_{11}^{11} & Z_{11}^{12} & Z_{12}^{11} & Z_{12}^{12} & Z_{12}^{13} \\
Z_{11}^{21} & Z_{11}^{22} & Z_{12}^{21} & Z_{12}^{22} & Z_{12}^{23} \\
Z_{21}^{11} & Z_{21}^{12} & Z_{22}^{11} & Z_{22}^{12} & Z_{22}^{13} \\
Z_{21}^{21} & Z_{21}^{22} & Z_{22}^{21} & Z_{22}^{22} & Z_{22}^{23} \\
Z_{21}^{31} & Z_{21}^{32} & Z_{22}^{31} & Z_{22}^{32} & Z_{22}^{33}
\end{array}\right]\left[\begin{array}{c}
I_{1}^{1} \\
I_{1}^{2} \\
I_{2}^{1} \\
I_{2}^{2} \\
I_{2}^{3}
\end{array}\right] .
$$

The coefficient $Z_{m k}^{\eta \mu}$ may appropriately be called the opencircuit transfer impedance from mode $\mu$ in waveguide $k$ to mode $\eta$ in waveguide $m$. If the reciprocity condition is satisfied, the above impedance matrix is symmetric, i. e. $Z_{m k}^{\mu \eta}=Z_{k m}^{\eta \mu}$

Washington, April 20, 1948. 